Journal of Al Azhar University Engineering Sector

Vol. 14, No. 52, July 2019, 1304-1317

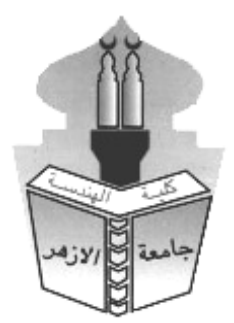

\title{
THE ROLE OF BIOMIMICRY IN DEVELOPING ARCHITECTURE DESIGN PROCCESS (CREATION \& DESIGN PROCESS)
}

\author{
Aya Aboelkhair, Mohammed Ezzat and Ahmed Fared Abdelfatah Saaid Hamza \\ Architecture Department, Faculty of Shoubra, Benha Universiy \\ avabashar237@amail.com, enpag 2003@yahoo.com, afh cairo@yahoo.com
}

\begin{abstract}
Humanity has recently witnessed a huge scientific uprise in all fields. This is by the virtue of scientific revolution that broke many classical rules and shattered the unjustifiable illusions as well as associating many entities. The concept of nature mimesis "Biomimicry" has its effect which inspired many thoughts in several fields. This concept has inspired architects all over the world. Consequently, new architectural trends appealed to be directed towards imitation thought which aimed to mimicking nature. However, application of the concept differs with the diversity of thoughts \& trends of each designer. Some adopt the application of form trend, others adopt the functional one, others make a harmonious combination the two parties \& others resort to the ecological systems. Because of that many manifestations \& trends have emerged. The combination of Biomimicry "Architecture of Nature", architectural strategies and creative strategies have led to a change in perception about the relationship between architecture and nature by breaking the restrictions of architectural features associated with Euclidean architecture which appeared in the modernity architecture. The need to preserve natural resources also necessitates a return to nature, and attention to natural forms, sciences and natural systems, and study living organisms that live in harmony and integration with their surroundings. The contemporary design relies on the use of digital and computer technology to simulate natural systems and to follow their forms, functional and structural characteristics that enable them to live in an optimal manner in order to achieve the dynamic and interactive relationship between architecture and the environment to achieve a dynamic balance between the various forms of life that govern natural systems. In this sense, it is important to study biomimicry as an input to the design process development and the extent to which architectural trends are influenced and taken into account in the design of buildings mimiking nature. Where the research seeks to reach an objective vision associating architecture to nature in a Creative Frames, we take advantage of our understanding of the interaction of organisms with the environment in a way that gives the balanced natural life of all organisms, thus achieving a dynamic balance between the different forms of life governed by natural systems and highlighting to such research in the development and improvement of the design of these buildings. Keywords: (Thinking, Creativity, Design Process, Architectural Space, the natural environment, Life's Genius, Biomimicry, Levels of Biomimicry, Voronoi algorithms, the architectural trends of Biomimicry).

Keywords: (Thinking, Creativity, Design Process, Architectural Space, the natural environment, Life's Genius, Biomimicry, Levels of Biomimicry, Voronoi algorithms, the architectural trends of Biomimicry).

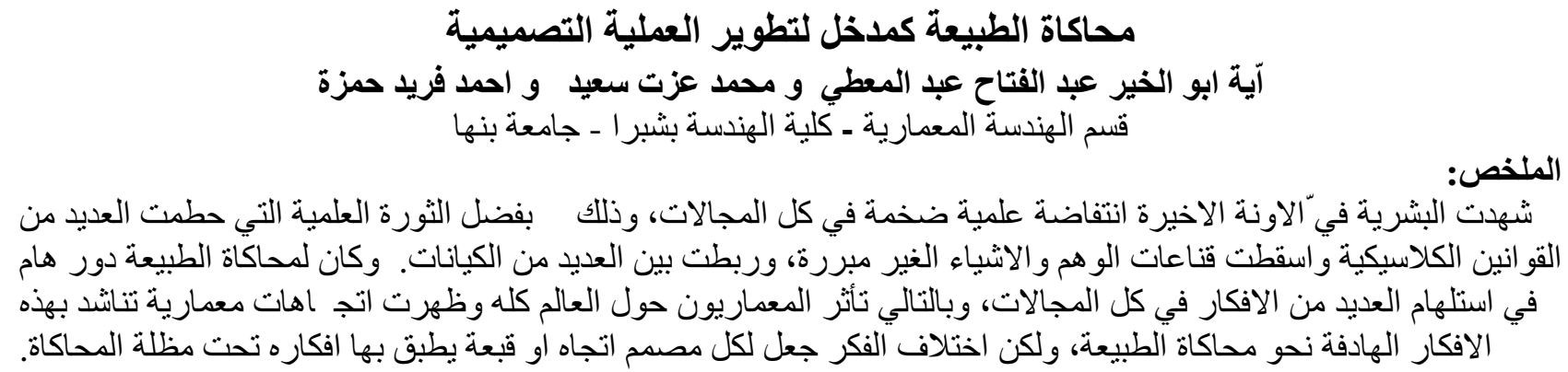


فالبعض اخد التطبيق الثكلي، واخرون الوظيفي، والبعض جمع بين الطرفين، و هناك من قرر ان يتخذ من الطبيعة بيئة كاملة

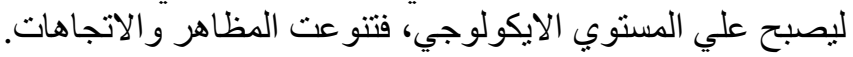

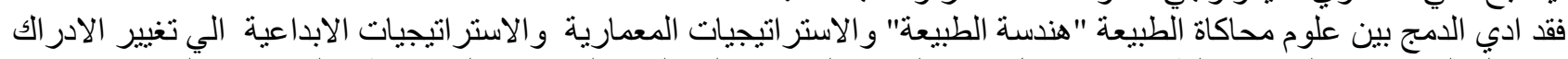

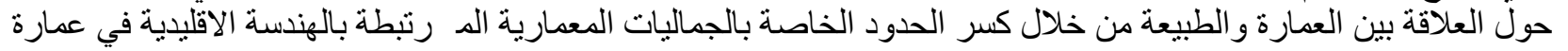

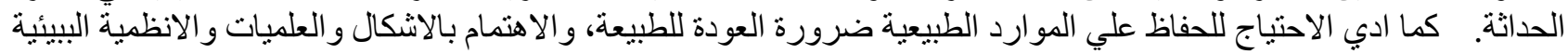

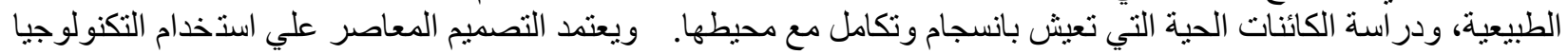

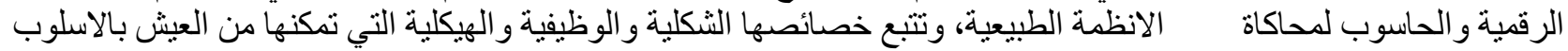

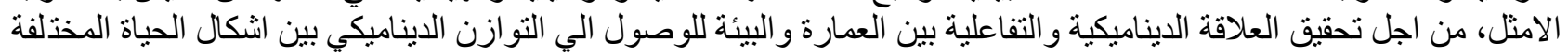
التي تحكم النظم الطبيعية.

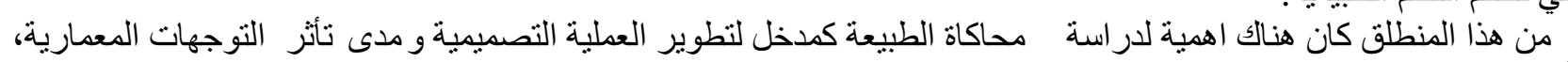

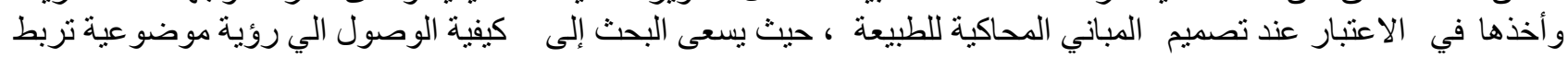

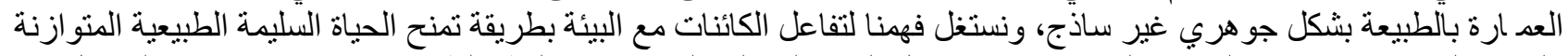

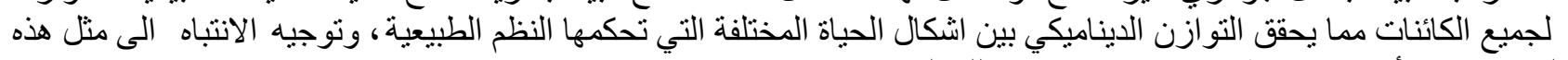

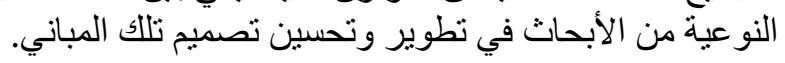

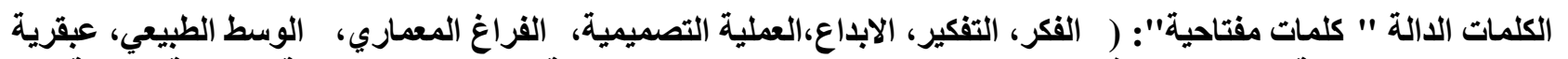

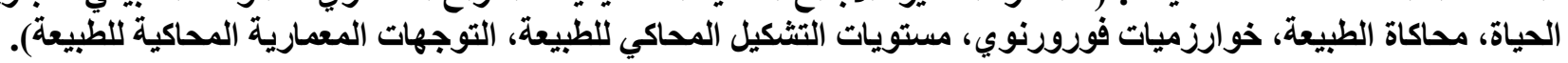

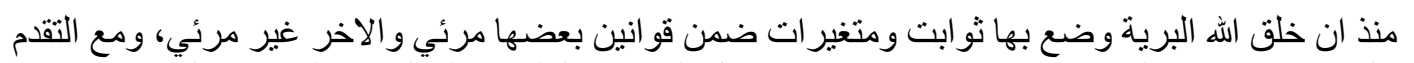

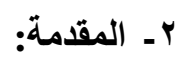

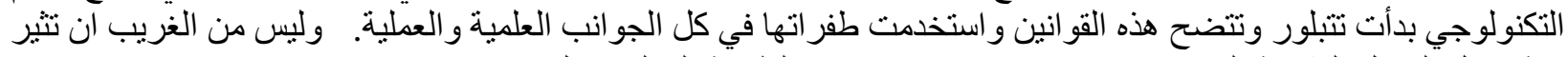

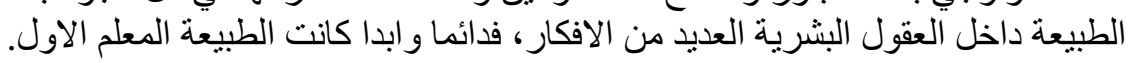

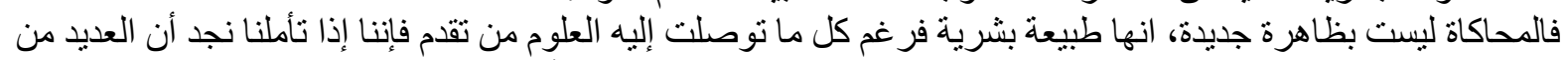

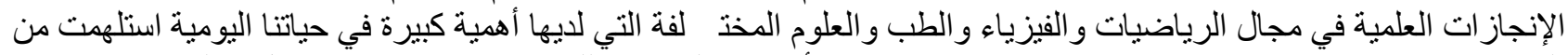

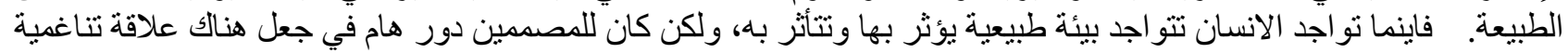
بين البيئة الطبيعية و الصناعية.

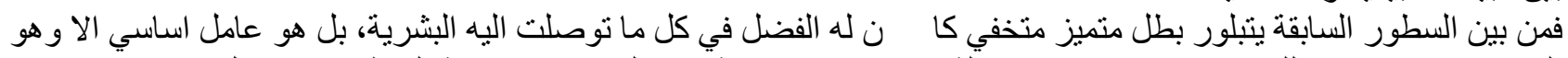

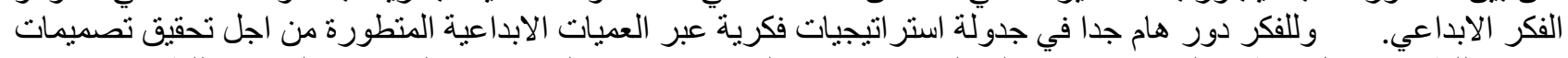

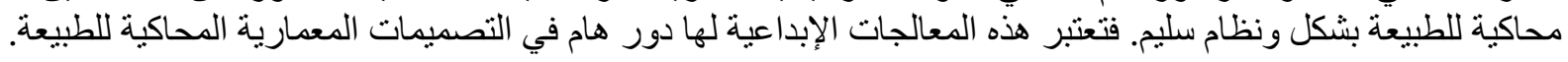

ץـ الإثكالية البحثية: تتمثل إثكالية هذه الدر اسة في محوريين رئيسيين هما:

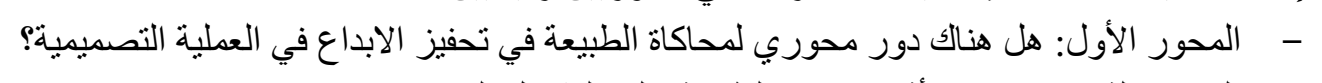

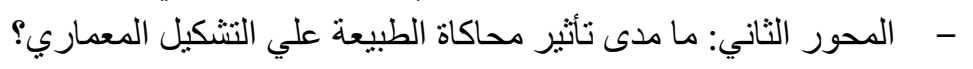

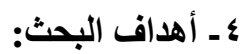

- - الوصول الى استر اتيجيات ابداعية في العملية التصميمية للمباني المحاكية للطبيعة.

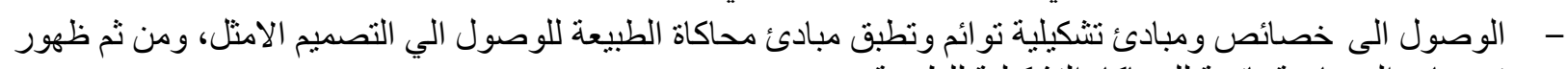
توجهات المعمارية ناتجة للمحاكاة التشكيلية للطبيعة.

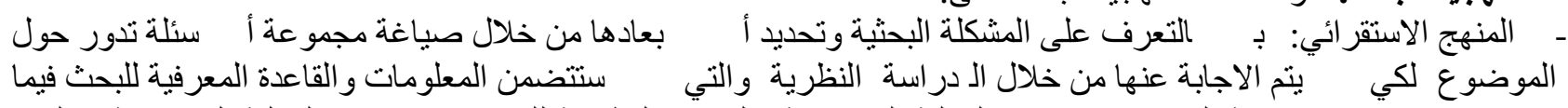
هـ منهجية البحث: سوف تعنمد منهجية البحث على:

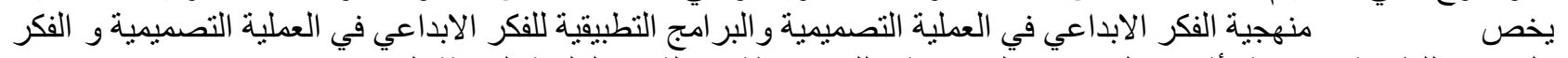

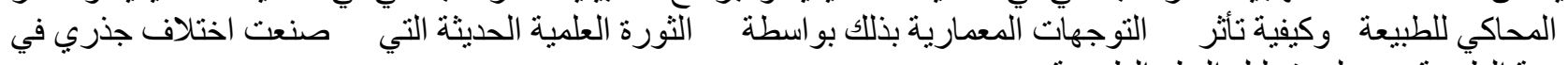
رؤية الطبيعة، وسهلت تحليل النظم الطبيعية.

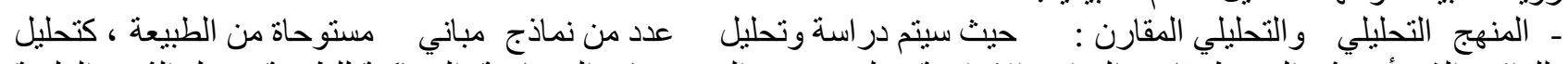

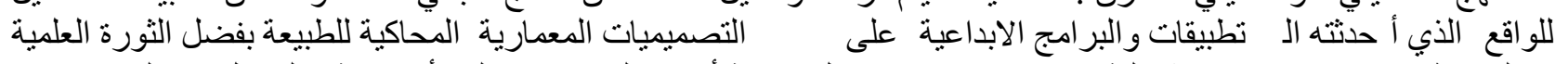

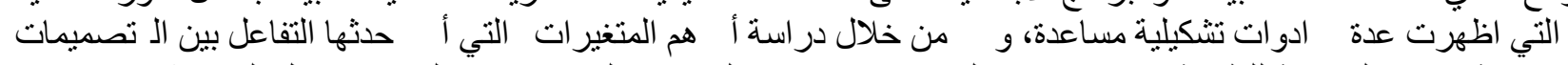

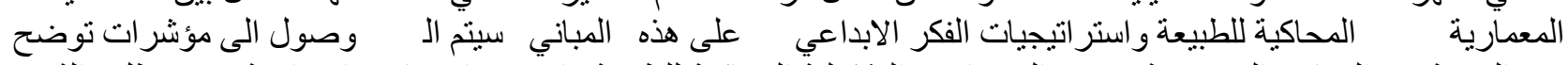

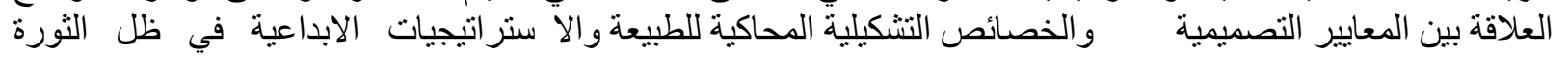


جـ الاراسة النظرية

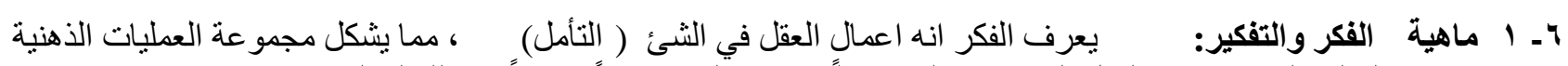

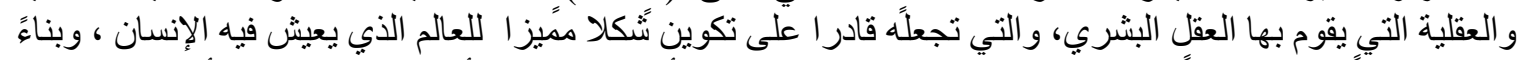

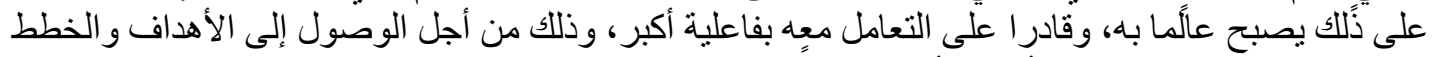

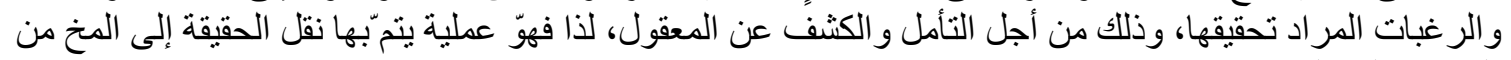
خلال استخدام الحواس المختلفة. التفكير فهو العملية العقلية التي يتم بموجبها حل المشكلات أو اتخاذ القرارات بطريقة علمية من خلال التفكير

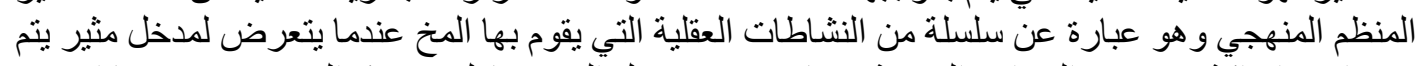

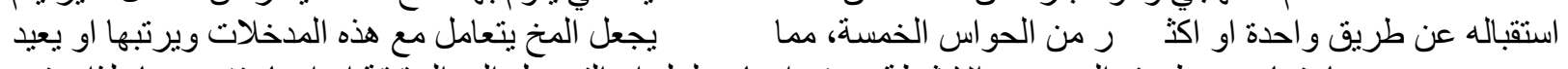

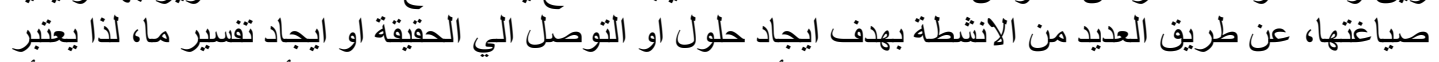

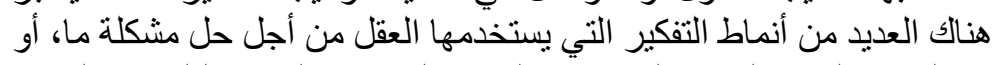
التفكير الناقد، التفكير المتقارب، التفكير المتباعد، التفكير التلقيدي، التفكير التفير التفير التفير

$$
\begin{aligned}
& \text { التفكير أعلي الوظائف الادر اكية. } \\
& \text { بغرض تفسير موقف ما، من هذه الادرية الانماط: } \\
& \text { المتشعب، و التفكير الجانبي، وغير هم العديد. }
\end{aligned}
$$

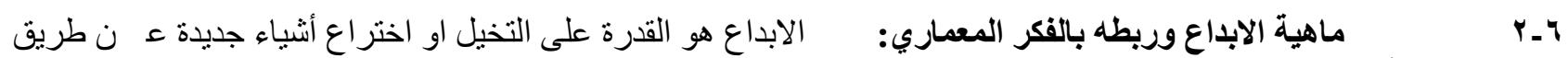

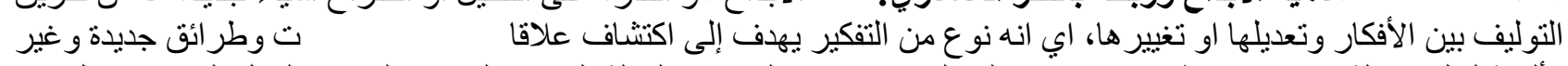

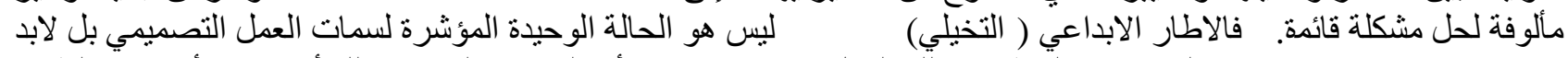

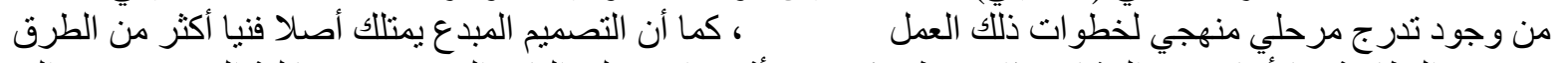

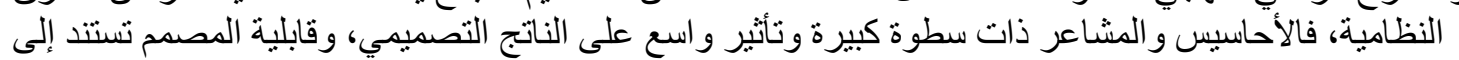

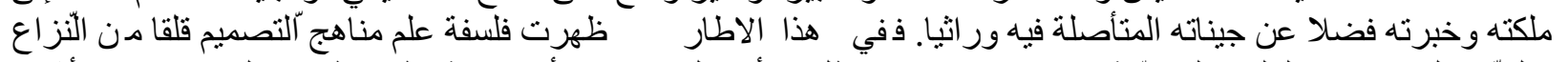

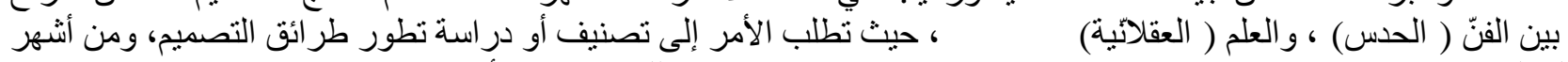

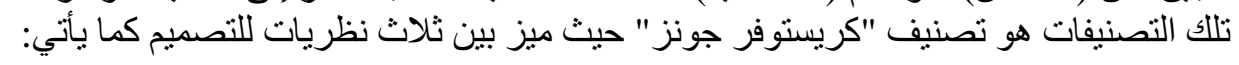

- المصمم كصندوق أسود Black box يمثل وجهة النظر الإبداعية حيث ينتج التصميم من الوثبة الإبداعية الغامضة.

- - المصمم كصندوق زجاج Glass box يمثل وجهة النظر المنطقية حيث ينتج التصميم من العملية المنطقية المبررة.

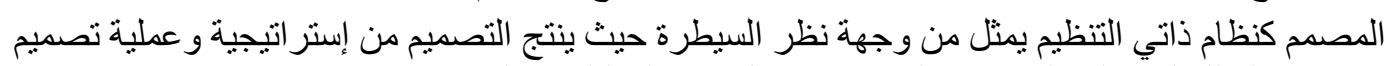
موضو عية، وتعتبر وسيلة للتغلب على التعارض الميطن من المود بين المنطق التحليلي، والتفكير الإبداعي".

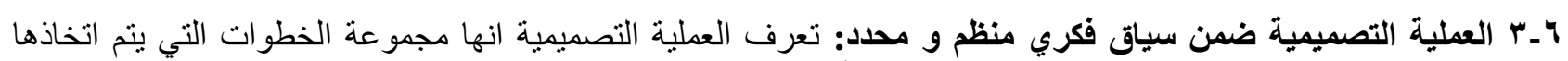

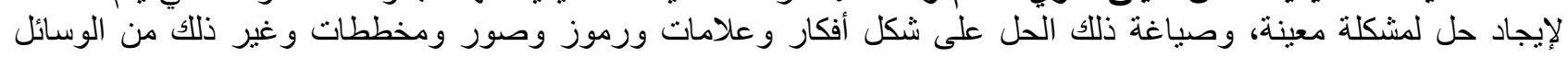

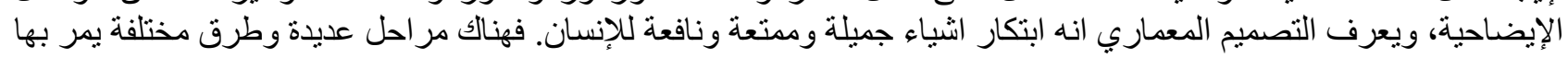

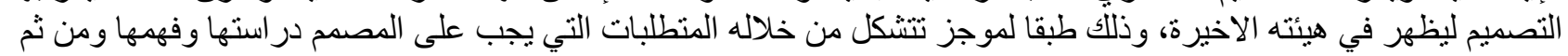

اختبار ها طبقا للمعايير التصميمية.

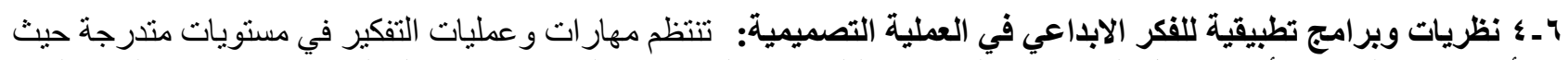

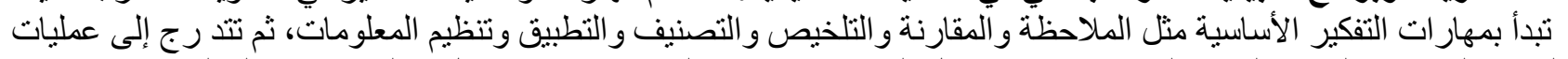

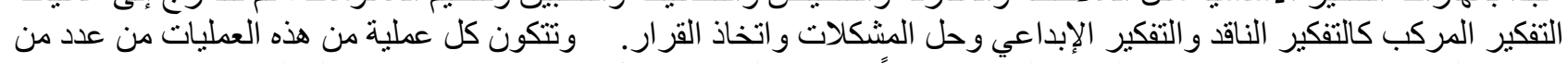

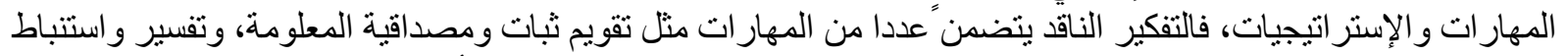

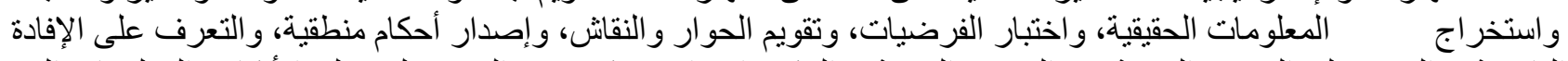

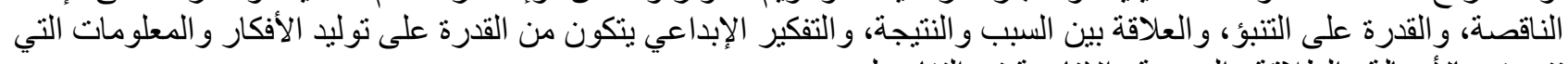

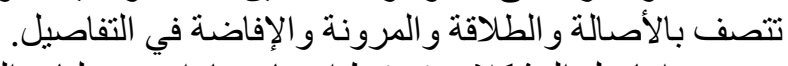

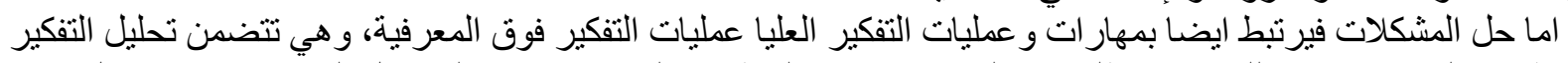

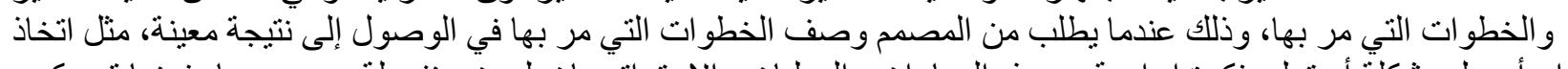

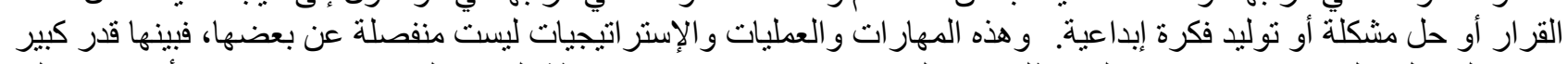

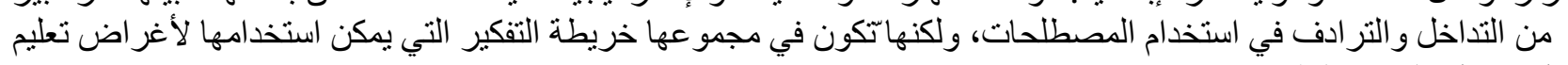
التفكير ومكوناته ومهار اته.

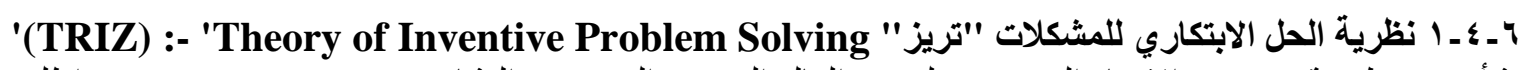

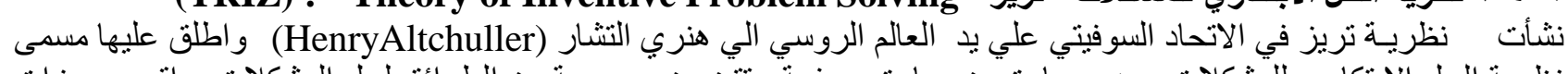

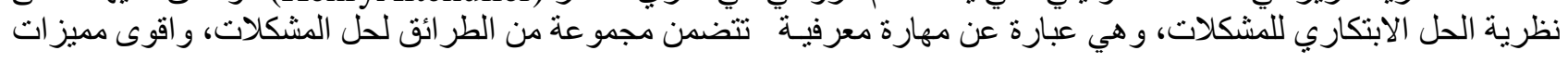


هذه النظرية انها لها قدرة على اجتياز العو ائق و المشكلات النفسية، ولها قدرت خارقة على تحليل العمليات لكي نستخدم المصادر

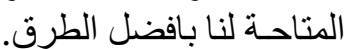

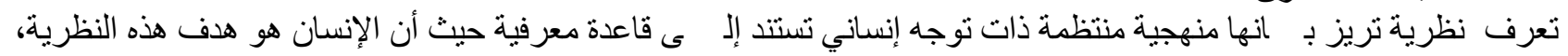
وتهدف إلى حل المشكلات بطريقة إبداعبة، وتثبر المنهجية إلى وجود نماذج عامة من النظم و العمليات ضمن الإطار العام لتحليل النظرية.

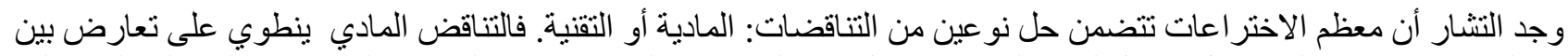

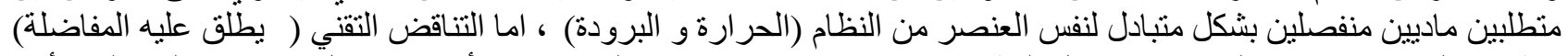

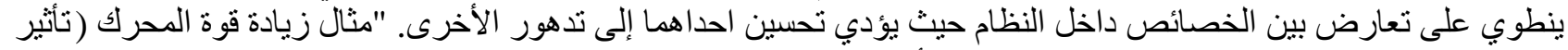

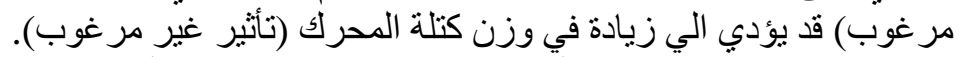

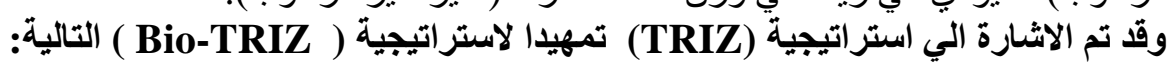

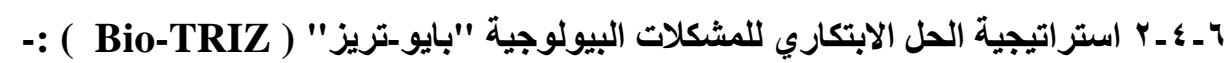

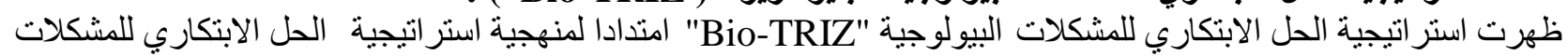

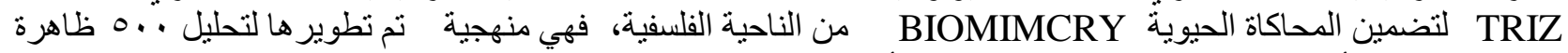

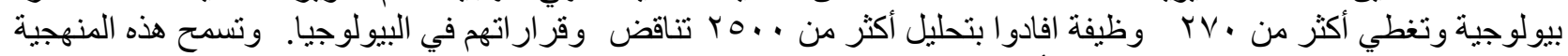

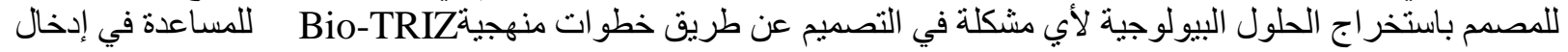

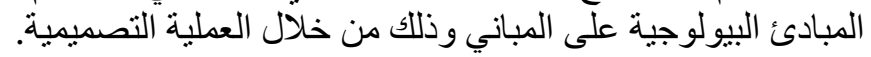

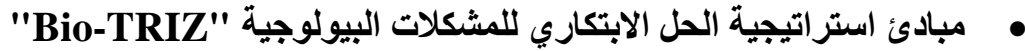

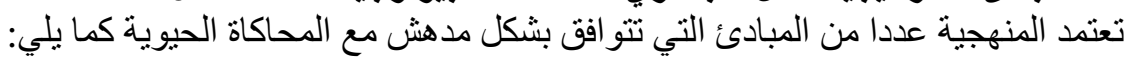

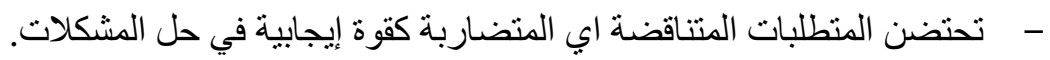

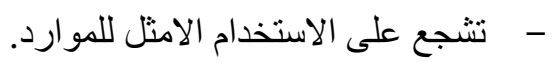 - - تعترف بالمحدودية "محدودية الموارد"، بما في ذللك تحويل العيوب إلى مو ارد مفيدة.

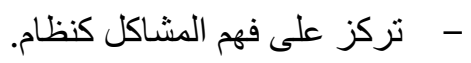 - - - - تسعي لتحديد الحل المثنالي.}

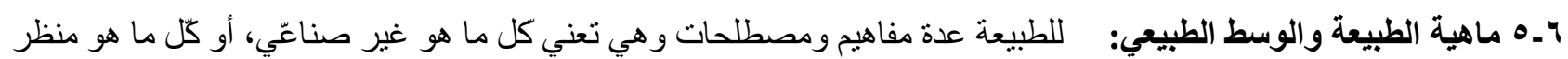

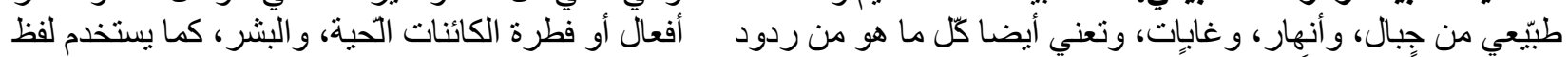

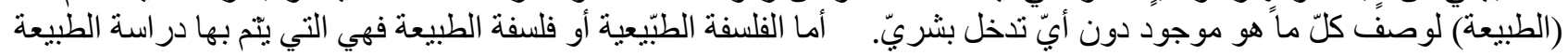

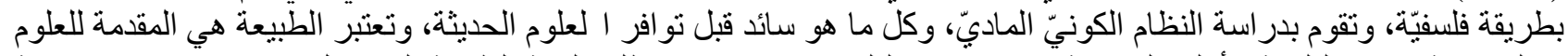

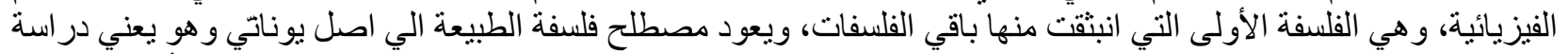

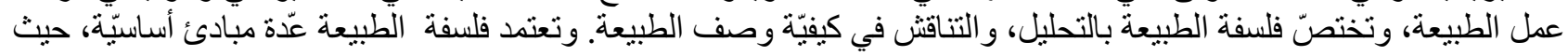

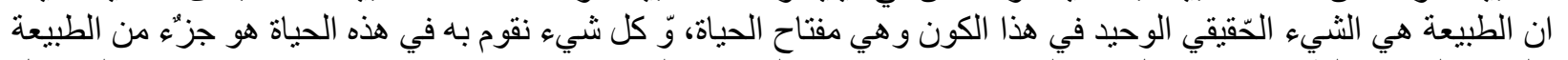

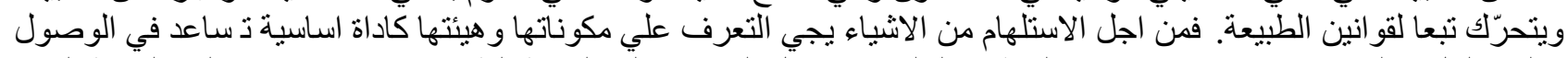

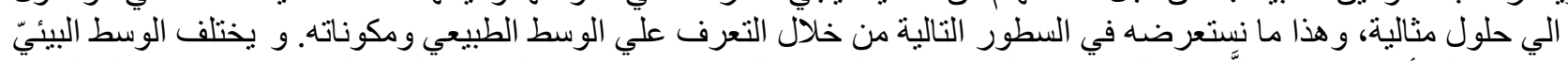

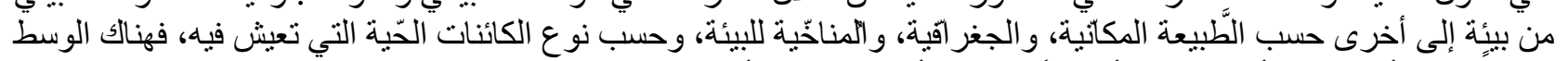

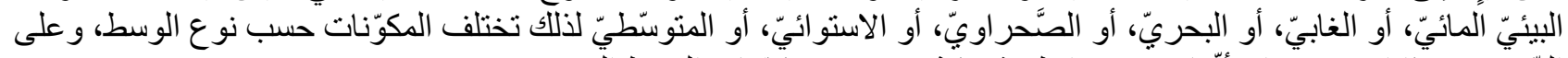

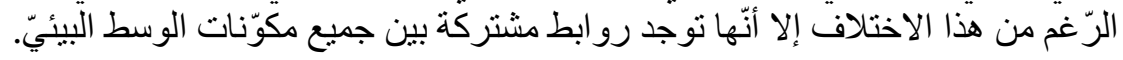

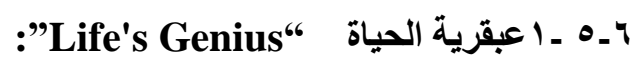

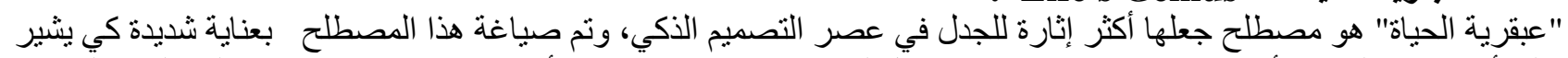

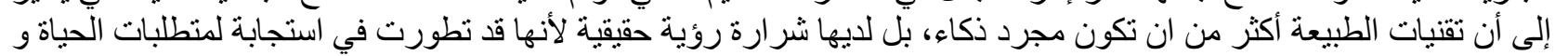

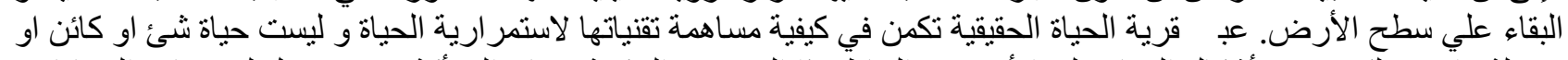

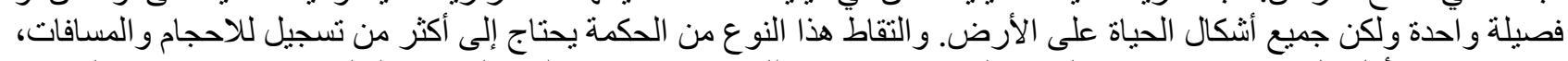

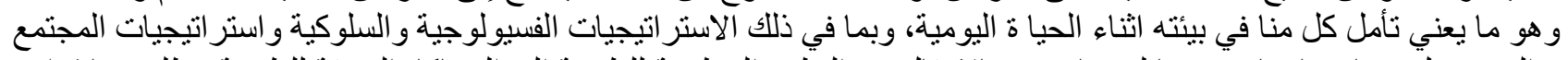

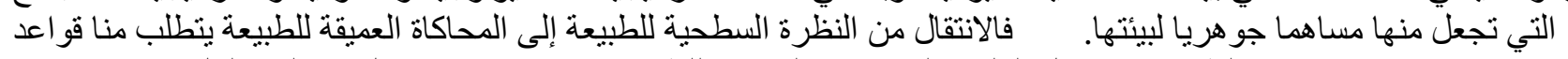

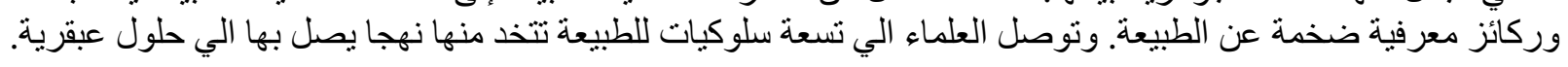


ד ـ 7 مفهوم محاكاة الطبيعة

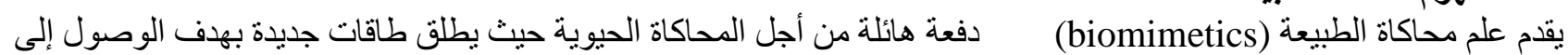

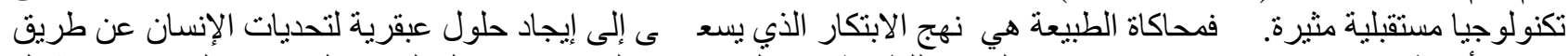

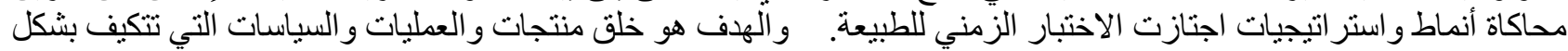

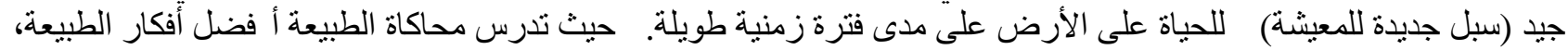

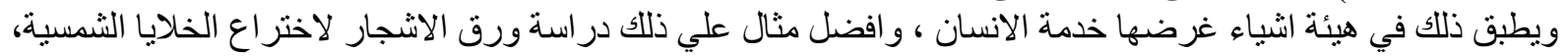

و افضل وصف لذللك بان "الابتكار ات مستوحاة من الطبيعة".

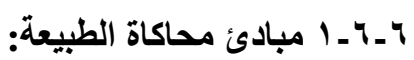

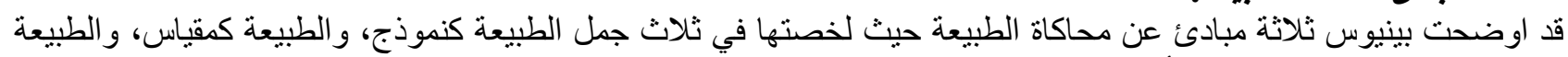

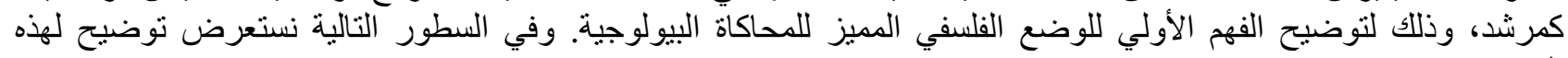

المبادئ.

- - مبدأ الطبيعة كنموذج: يوحي بمحاكاة طريقة الطبيعة لإحضار الأشباء اي لصنع الوظائف "الطبيعة تتناسب مع وظبيفتها".

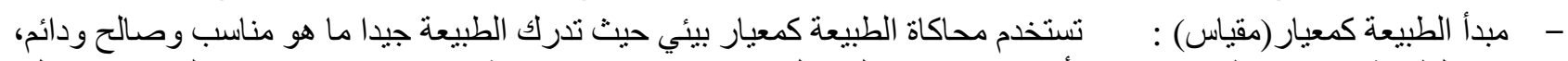

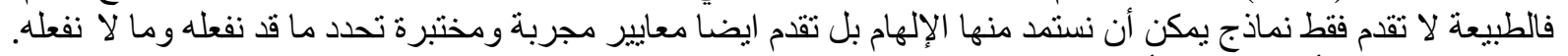

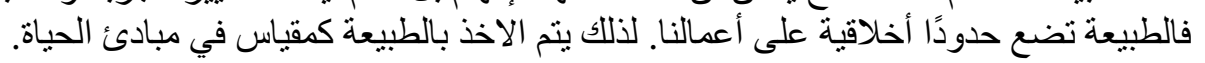

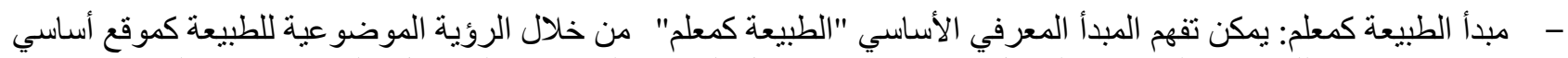

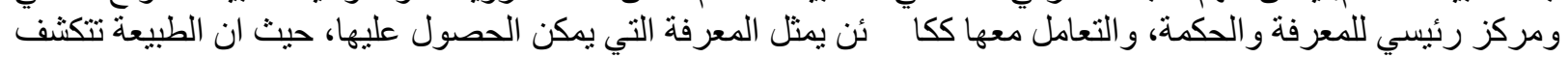

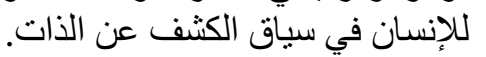

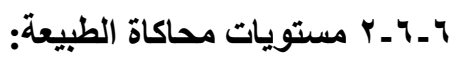

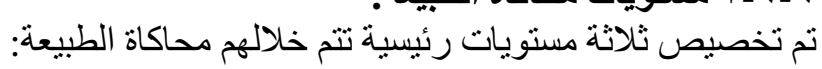

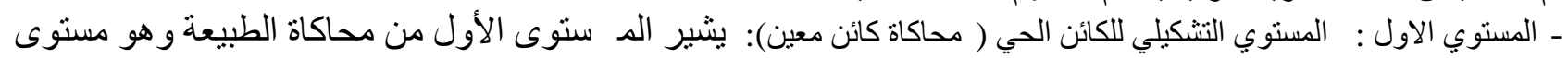

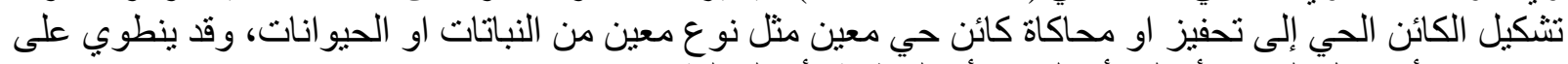

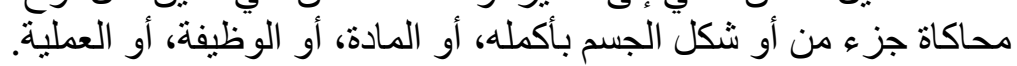

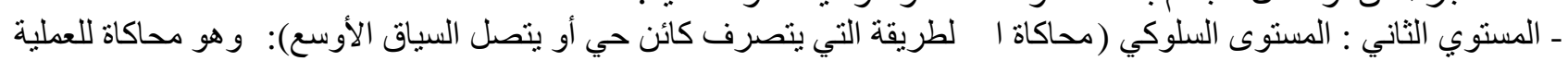

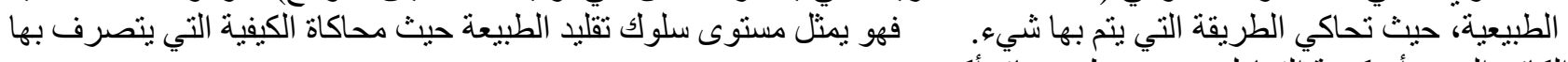

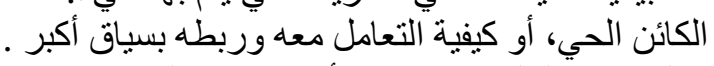

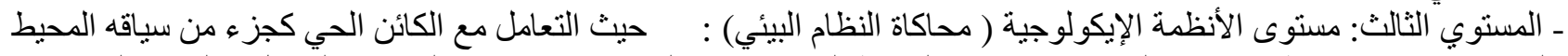

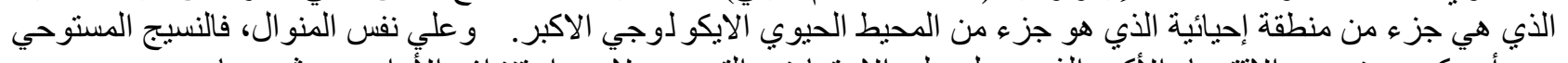

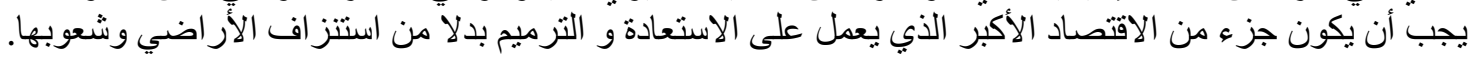

V-7 عمارة محاكاة الطبيعة:

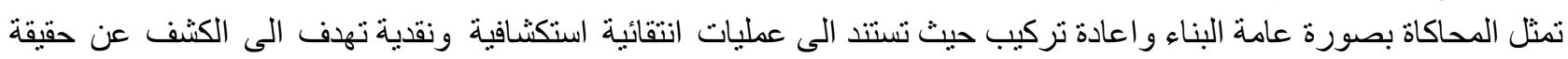

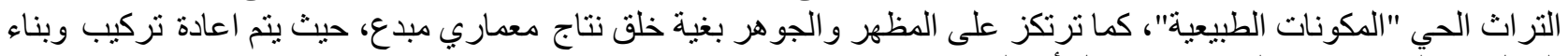

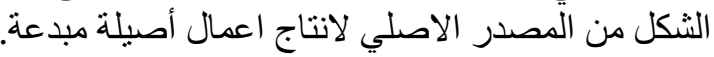

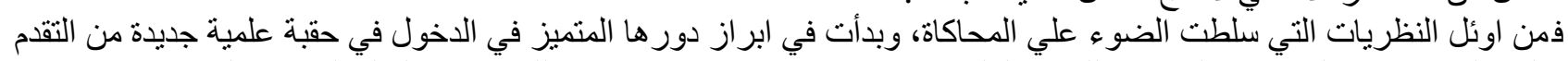

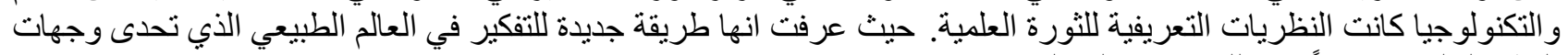

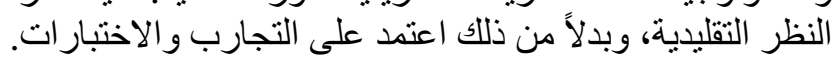

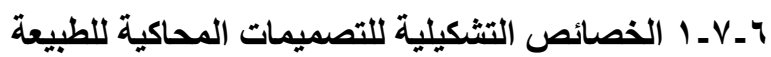

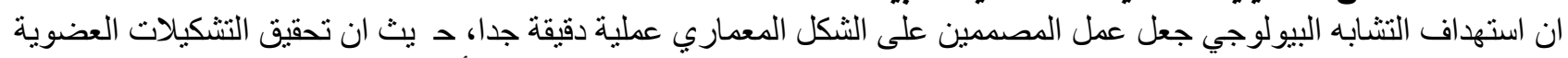

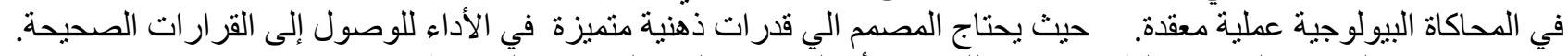

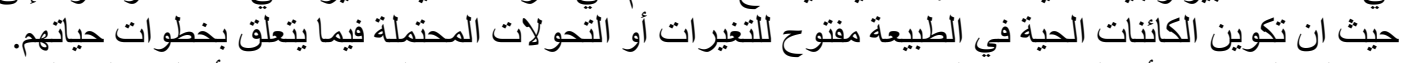

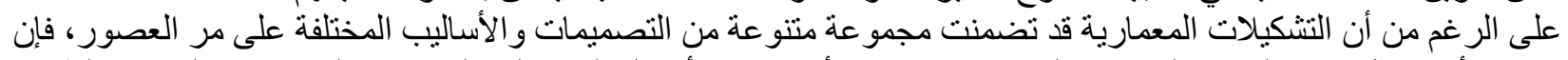

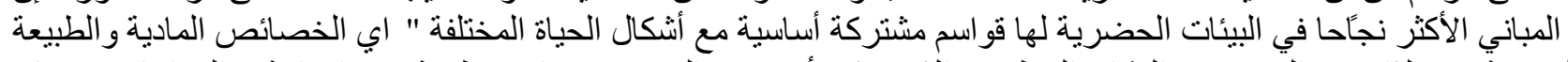

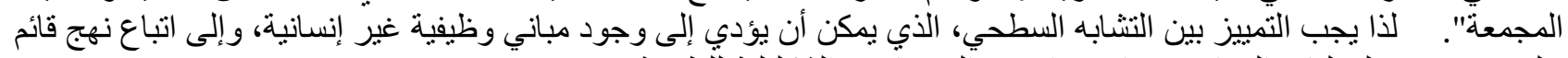

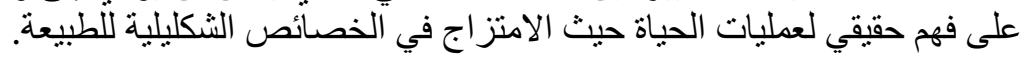


خوارزميات فورونوي كأداة تثكيلية في التصميم المحاكي للطبيعة

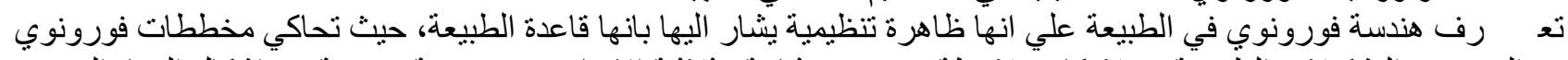

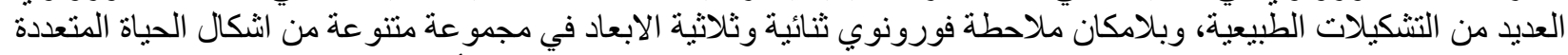

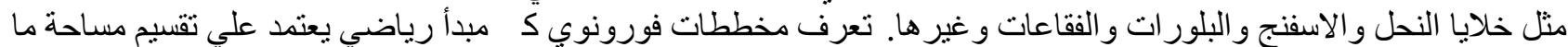

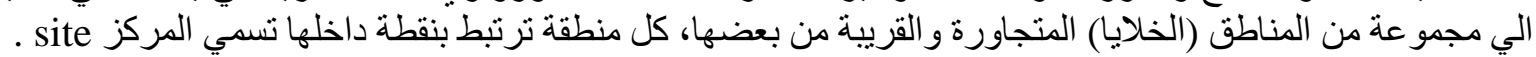

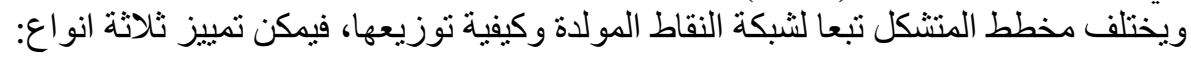

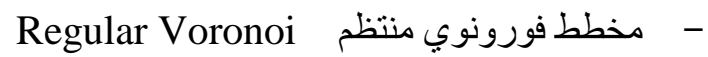

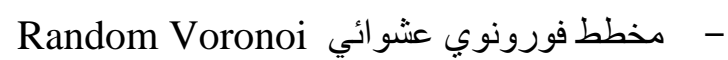
clustered Voronoi مخطط فورونوي تجميعي عis -

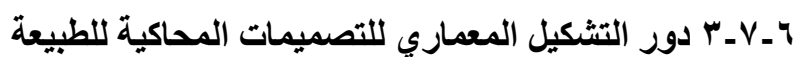

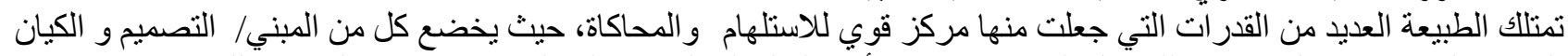

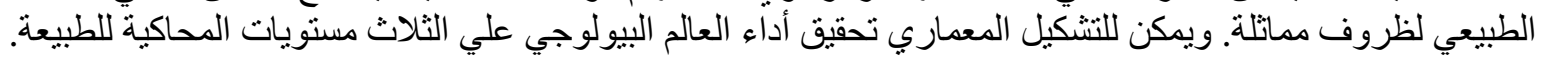

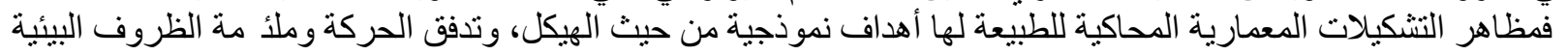

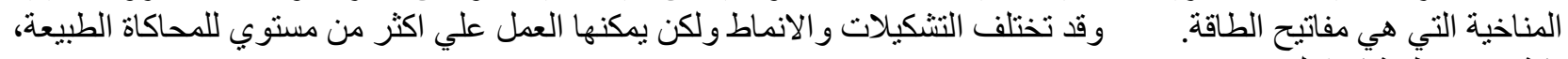

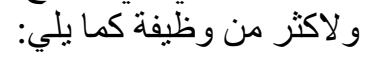

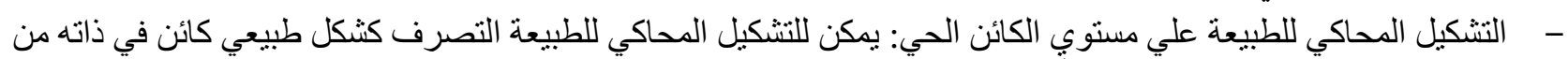

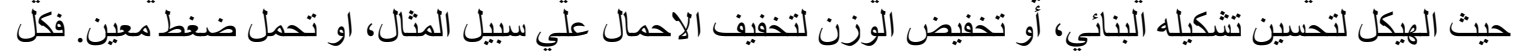

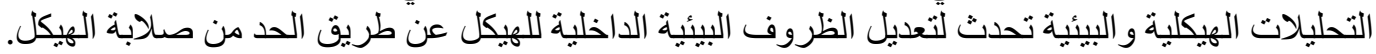

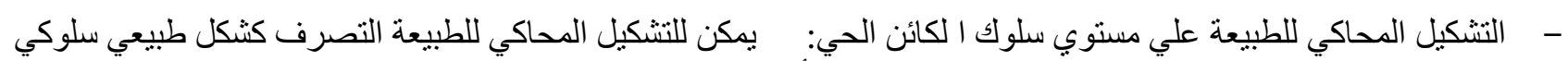

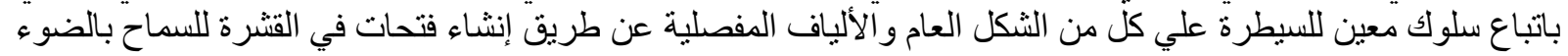

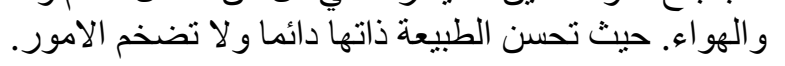

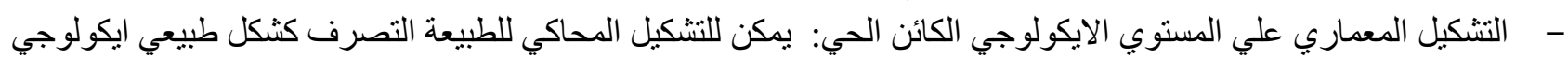

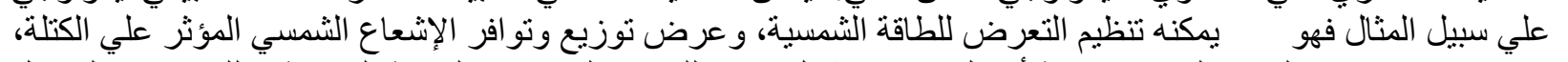

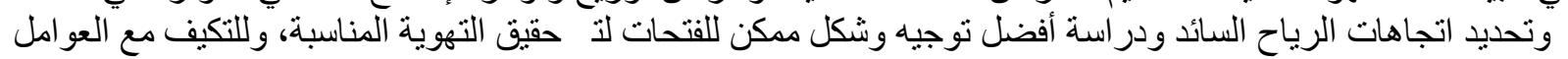

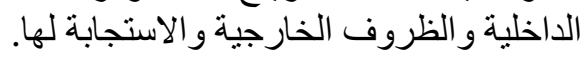

V - الدراسة التحليلية

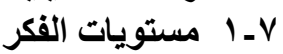
ثلاثة مستويات من تعريفات الفكر، فيما يلي الفكر

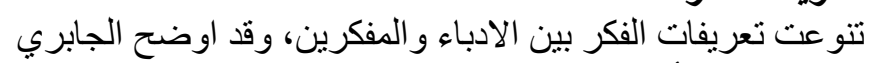
جدول (1) مستويات الفكر للجابري. المصدر: الجابري، ، 199 كأيديولوجيا، و الفكر كأداة، و الفكر كمحتوى:

\begin{tabular}{|c|c|c|}
\hline الفكر كمحتوى & الفكر كأداة & الفكر كأيديولوجيا \\
\hline 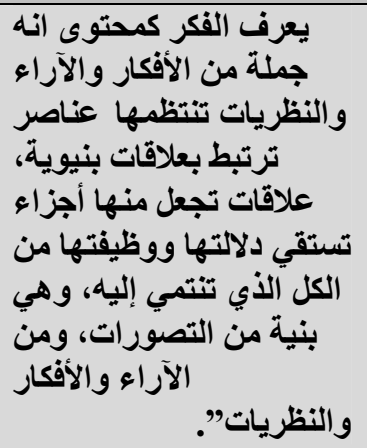 & 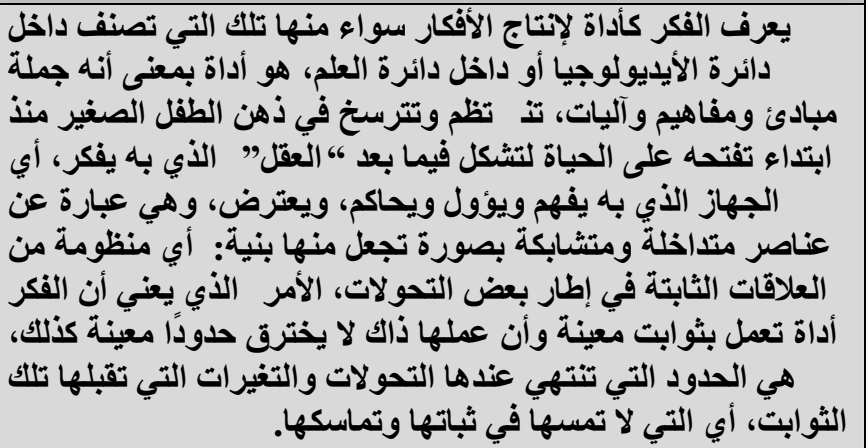 & 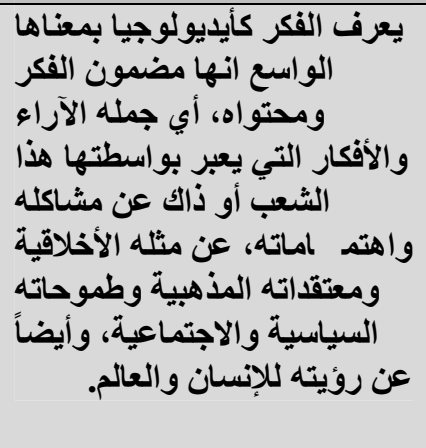 \\
\hline
\end{tabular}


جدول (ץ) تعريف التفكير المتثعب وربطه بالابداعـ المصدر: الباحث

\begin{tabular}{|c|c|}
\hline \multicolumn{2}{|l|}{ • Divergent Thinking التفكير المتشعب } \\
\hline 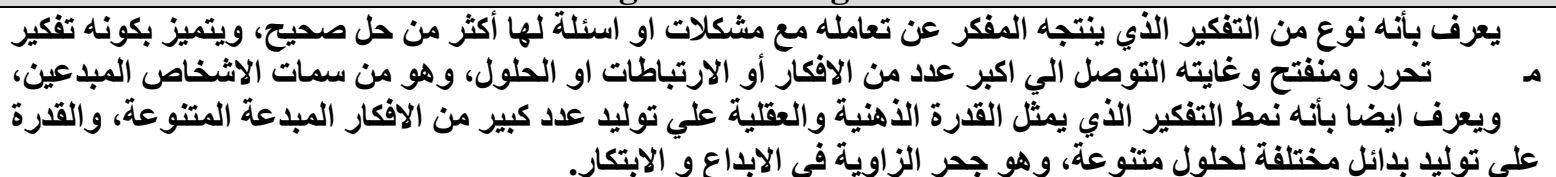 & التعريف \\
\hline 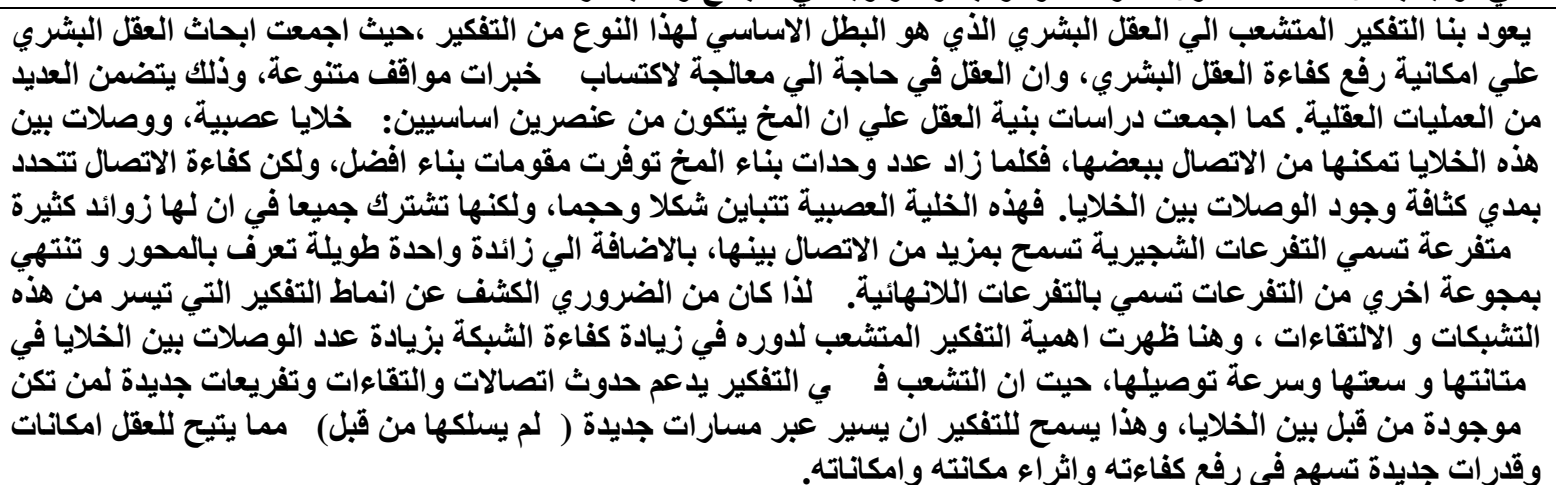 & بالتفكير \\
\hline
\end{tabular}

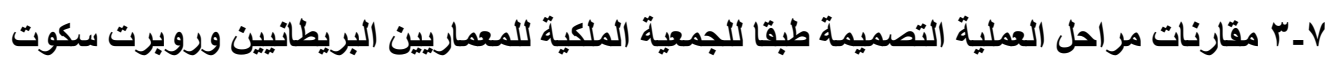

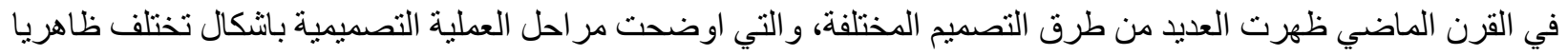
وتتشابه ضمنيا، وفيما يلي مر احل العملية التصميمية طبقا للجمعية الملكية للمعماريين البريطانيين (RIBA) :

Pدول (") مراحل العملية التصميمية طبقا للجمعية الملكية للمعماريين البريطانيين - Divis, 2010

\begin{tabular}{|c|c|}
\hline 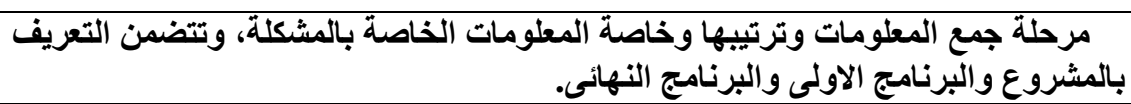 & جمع المطلومات Assimilation \\
\hline و التقيع. دراسة عامة و استكشَف طبيعة المشكلة والحلول المتاحة وطرق الحل وايجاد الاشكال & دراسة عامة General Study \\
\hline تطوير الحل او الحلول المختارة خلال الخطوة الثانية. & تطوير الحل Development \\
\hline توصيل الحل او الحلول للاشخاص داخل او خارج فريق العمل. & توصيل الحل Communication \\
\hline
\end{tabular}

وفيما يخص مر احل العملية التصميمية فقد صاغ روبرت سكوت مر احل العملية التصميمية كما يلي:

جدول (๕) مراحل التصميم المعماري طبقا لروبرت سكوت. المصدر: سكوت، . 190

\begin{tabular}{|c|c|}
\hline 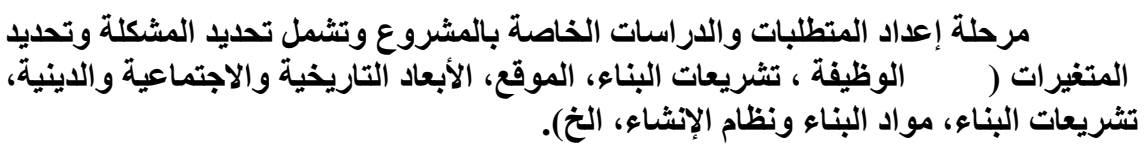 & $\underline{\text { Data Collection Briefing Stage }}$ \\
\hline 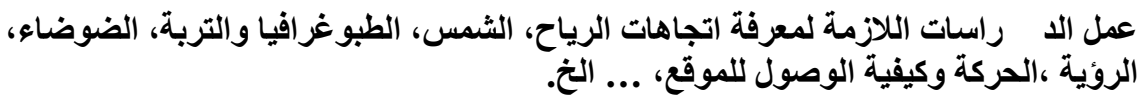 & Data Analysis تحليل المعلومات \\
\hline تحديد عناصر المشروع، وضع مخطط العلاقات الوظيفية. & تولا الفكرة \\
\hline رسم المساقط وتحديد الثكل الأولي. & وضع الحلول الأوليةة \\
\hline إجراء التقييم وتطوير الحل المناسب. & Evaluation مقارنة و تقييم الحلول \\
\hline
\end{tabular}

فى كل خطوة من هذه الخطو ات فان المشاكل التى يجب حلها تتطلب من المصدم تنسيق حلول للمشاكل الاكثر فاعلية. و هنالك العديد

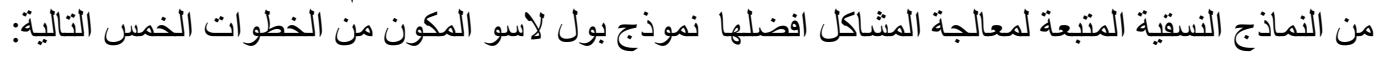


جدول (0) نموذج بول لاسو لحل المشكلات- المصدر: Laseau, 1980

\begin{tabular}{|c|c|}
\hline 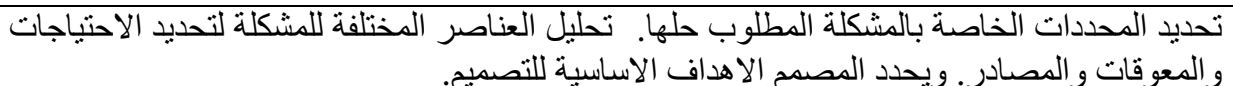 & تعريف المشكلة Problem Definition تعل \\
\hline يقوم الدصمم باختبار الحلول المتوافرة و الجديدة ويقوم بتطوير عدد من المر ادفات المقبولة. & 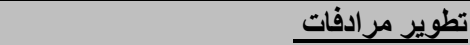 \\
\hline & Developing Alternatives \\
\hline ليتنم تبنى عدد من المعايير للتقيبم بناء على اهداف التصميم. ويتم ترتيب مر ادف ات الحل حسب معايير & Evaluation التقيّي \\
\hline 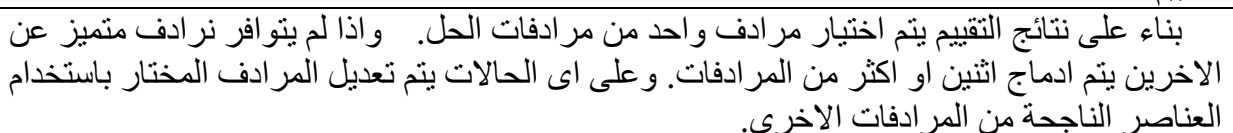 & Selection \\
\hline الحل النهائى للمشكلة يجب ان يتم وصفه بطريقة تجعله قابل للاستخدام فى المرحلة التالية من التصميم & الاتصال Communication \\
\hline
\end{tabular}

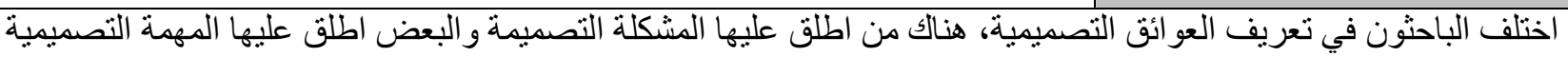
"Design Task"

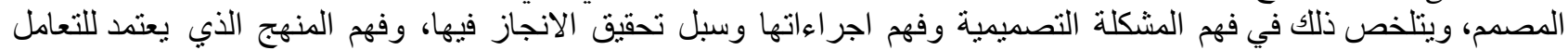

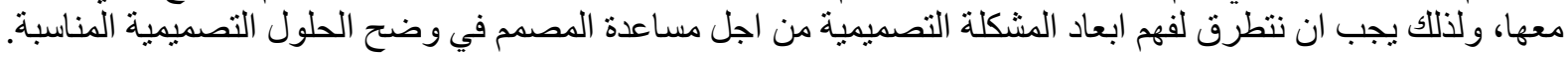

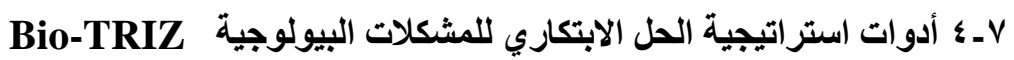

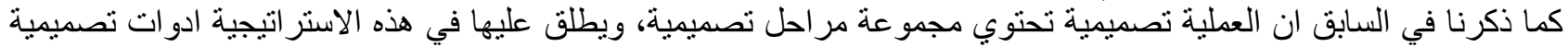

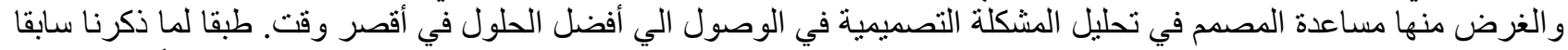

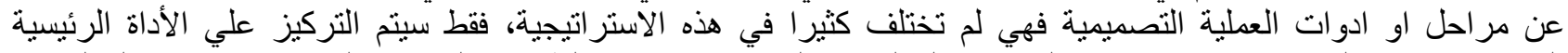

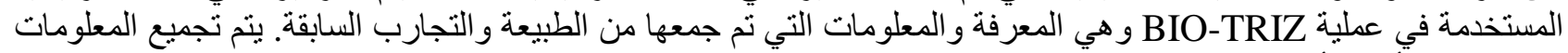
وجدولتها في أربعة أقسام:

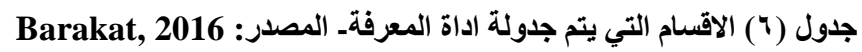

\begin{tabular}{|c|c|}
\hline \multirow{2}{*}{ 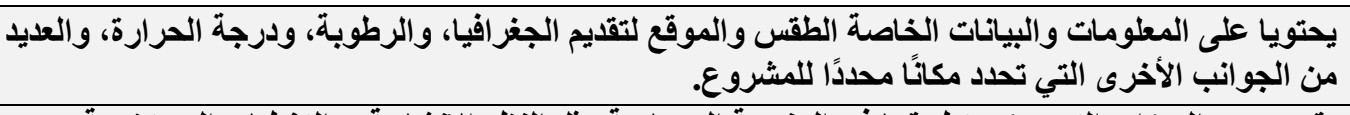 } & القسم الأول \\
\hline & القسم الثاني \\
\hline 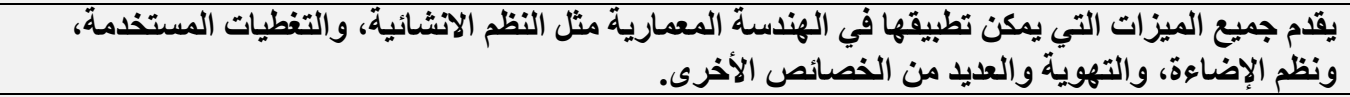 & القسم الثالث \\
\hline كل ميزة. القسم الرابع جميع الوسائل والحلول المشتقة من الحيوانات والنباتات والطبيعة، والتي يمكنها تحقيق & القسم الرابع \\
\hline
\end{tabular}

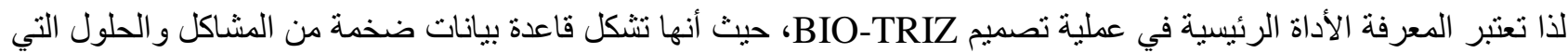
تقوم بها العملية. هنالك اربعة مر احل لاستخدام هذه الأداة حيث يتم تمر هذه البيانات علي المر احل التهان التالية:

جدول (Varakat, 2016 - BIO-TRIZ مراحل استخدام اداة المعرفة في عملية تصميم

\begin{tabular}{|c|c|c|}
\hline يتم فيها تحليل المشكلة بثكل منظم جدًا. & مرحلة التحليلAnalyze phase مريل & - \\
\hline يتم فيها استخراج كل الحلول المكنة من الطبيعة. & مرحلة الاستخراجExtract phase & - \\
\hline في شئم فيها فصل الحل لكل مشكلة حيث يتم تعيين الحلول المناسبة لكل مشكلة ويتم جمعها & مرحلة الفصل Judge Phase & - \\
\hline حيث تتم مقارنة الحلول المختارة معًا ويتم استخراج النموذج الأمثل واختباره. & مرحلة الاختبار Testing Phase & - \\
\hline
\end{tabular}

تنتج من منهجية BIO-TRIZ "التي تعتمد على قاعدة بيانات ضخمة من الميز ات التهي التي يمكن نطبيقها على المباني، و على منهجية

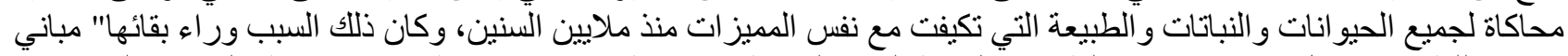

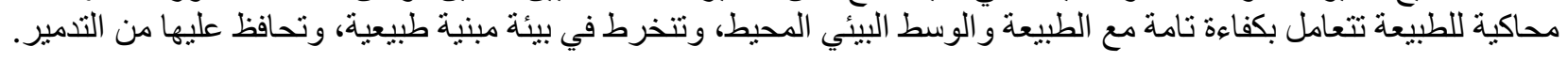

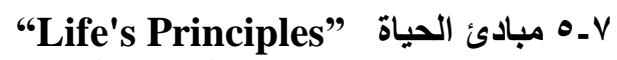

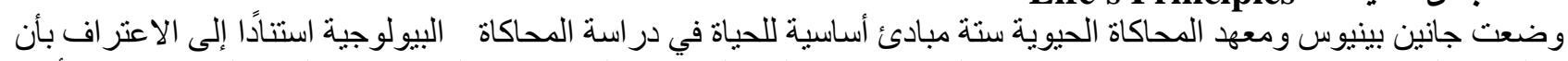

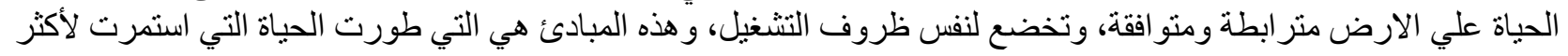




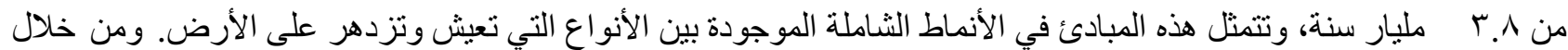

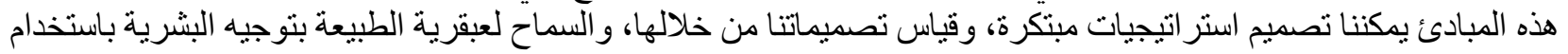
مبادئ الحياة كمبادئ لطموحاتنا المستقبلية. وتتمثل هذه المبات المبادئ في يلي:

جدول (^) مبادي الحياة ـالمصدر: Biomimicry Institute 3.8

\begin{tabular}{|c|c|}
\hline طرق التطبيق & المبادئ \\
\hline 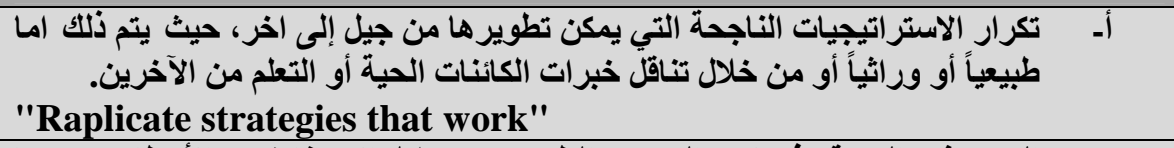 & 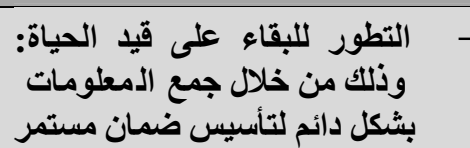 \\
\hline 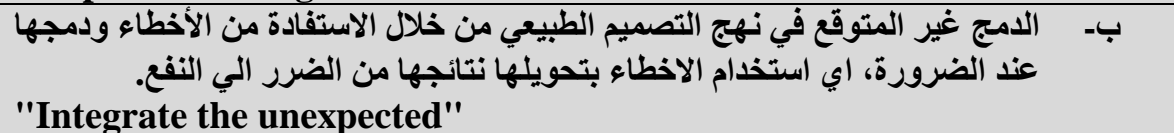 & "Evolve to survive" \\
\hline 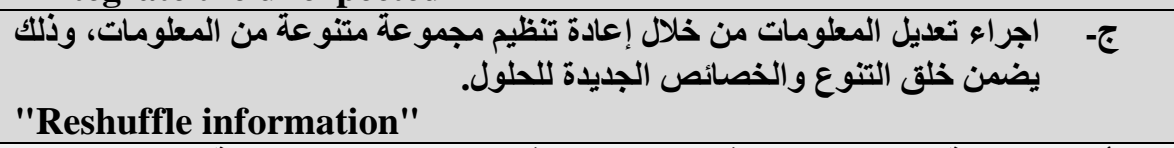 & \\
\hline 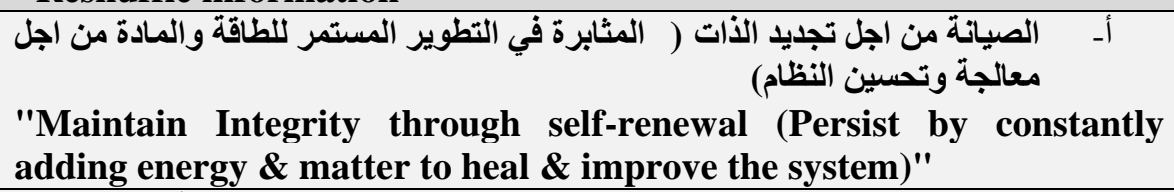 & 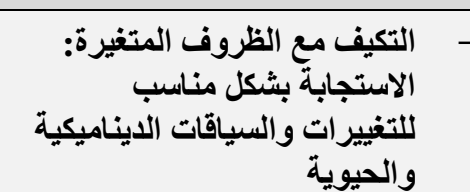 \\
\hline 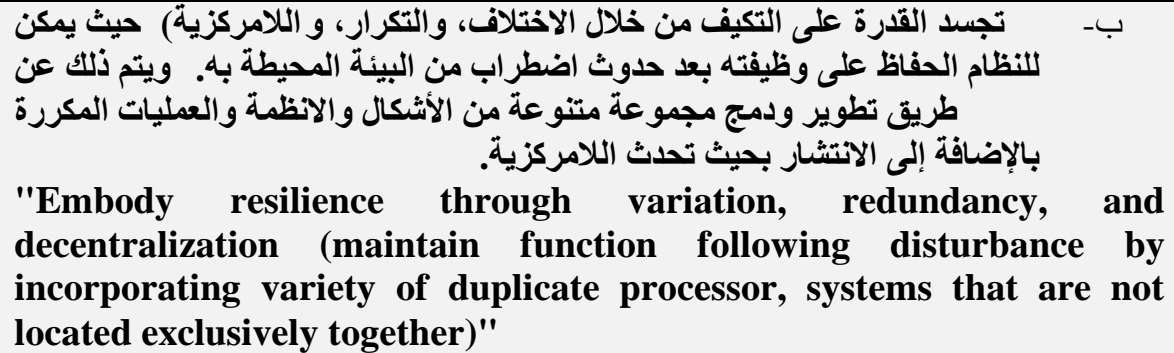 & $\begin{array}{l}\text { "Adapt to changing } \\
\text { coniditions" }\end{array}$ \\
\hline $\begin{array}{c}\text { ج- الامج المتنوع بين العديد من الاشكال والعمليات والوظائف لتلبية الإحتياجات. } \\
\text { "Incorporation Diversity" }\end{array}$ & \\
\hline $\begin{array}{l}\text { أ- استخام المواد والطاقة المتاحة بسلاسة (الاستفادة المثلي من المواد والطاقة) } \\
\text { "Use readily available materials \& energy (Build with abundant, } \\
\text { accessable materials while harnessing freely avaible energy)" }\end{array}$ & 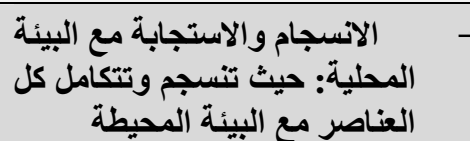 \\
\hline 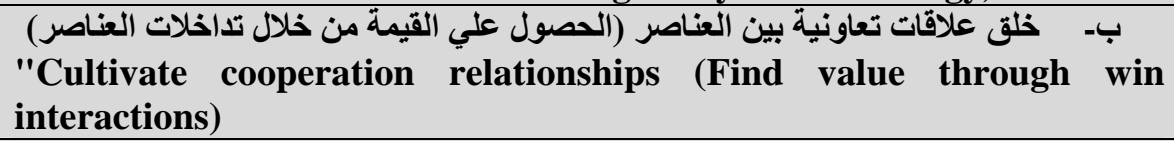 & $\begin{array}{l}\text { "Be locally attuned \& } \\
\text { responsive" }\end{array}$ \\
\hline 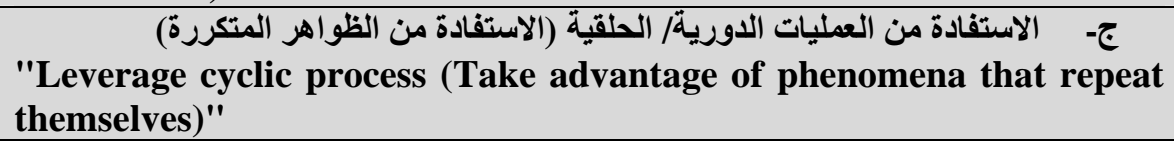 & \\
\hline 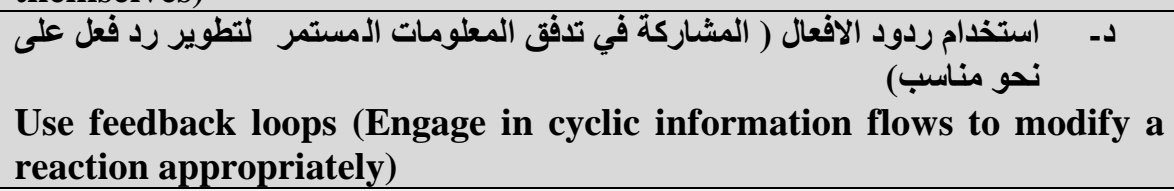 & \\
\hline أ- البناء الانتقائية للنماذج المختارة من العناصر (حيث التشكيل الابداعي المميز للعناصر & العملات التخدام الكيمياء التي تدعم \\
\hline $\begin{array}{l}\text { "Build selectively with a small subset of elements "(assemble } \\
\text { relativelyfew elements in elegent ways)" }\end{array}$ & $\begin{array}{l}\text { "Use life-friendly } \\
\text { Chemistry" }\end{array}$ \\
\hline 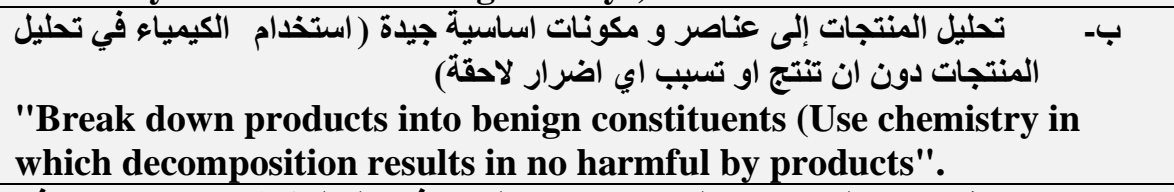 & \\
\hline 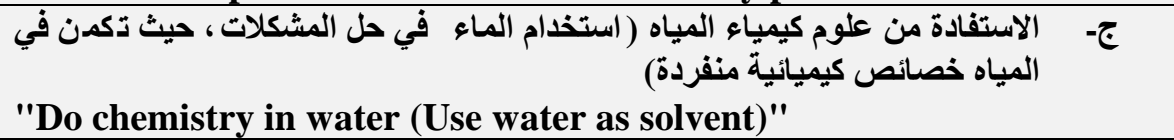 & \\
\hline
\end{tabular}




\begin{tabular}{|c|c|}
\hline 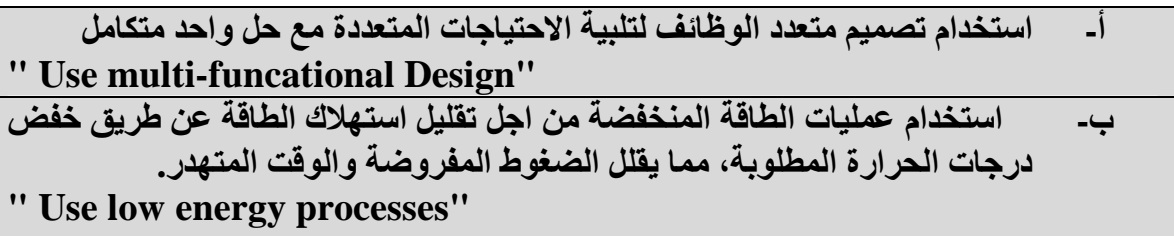 & \multirow{3}{*}{ - مفاءة الموارد (المادية والطاقة): } \\
\hline "Recycle all materials" اعادة تدوير جميع المواد (إبقاء كل المواد في حلقة مغلقة) & \\
\hline 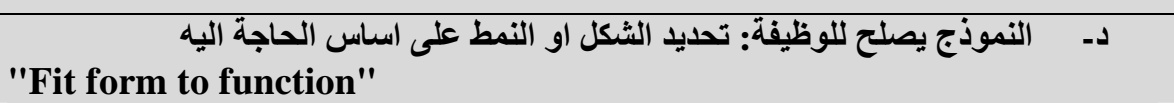 & \\
\hline 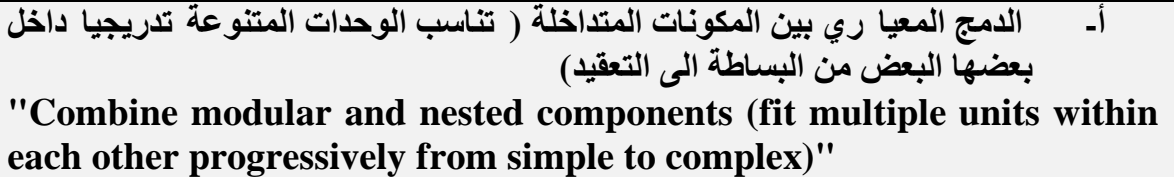 & \multirow{3}{*}{ 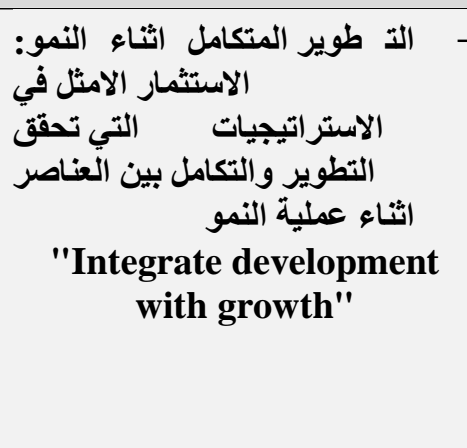 } \\
\hline 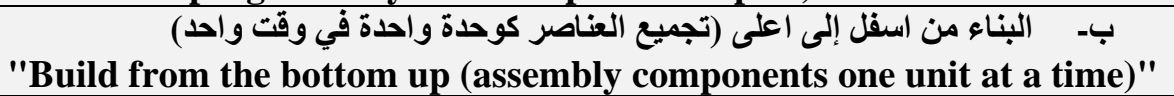 & \\
\hline 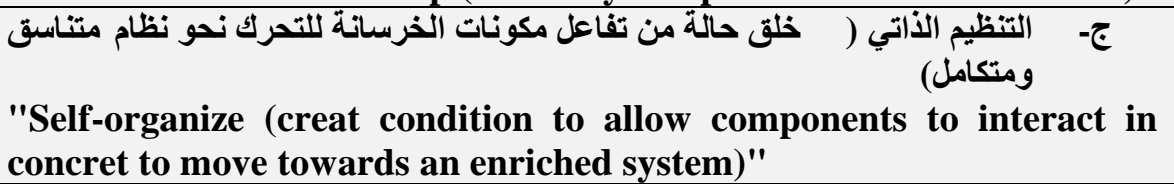 & \\
\hline
\end{tabular}

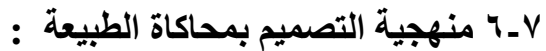
تتصنف منهجية التصميم بمحيماكاة الطبيعة الي فئتين منهجية مبانشرة، منهجية غير مباثرة، ولكن معهو محاكاة الطبيعة و المتعاونين

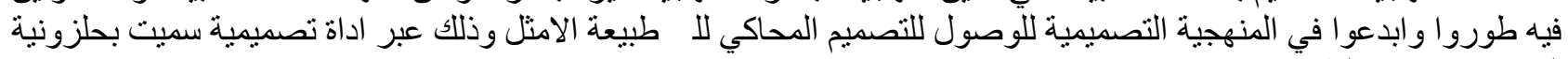

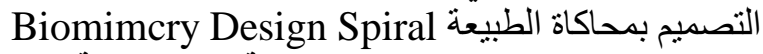
جدول (9) مقارنة بين المنهجية المباشرة والغير مباشرة ـ المصدر: McHarg, 1995.

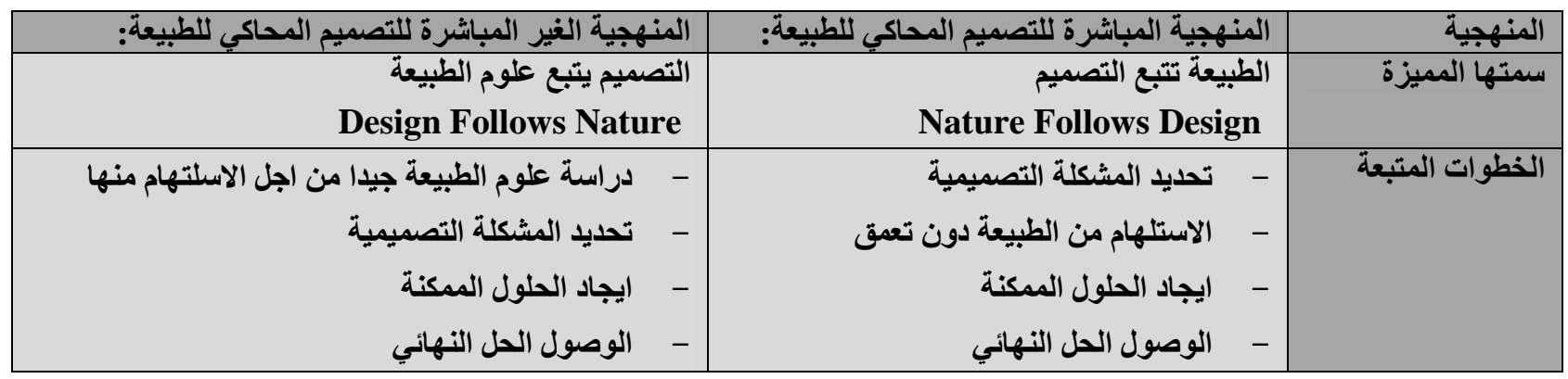

Vل حزونية التصميم بمحاكاة الطبيعة: V-V

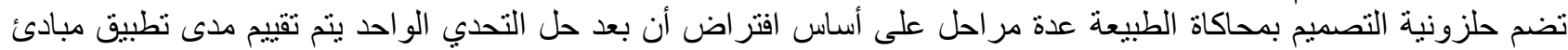
الحياة، ثم نلجأ غالبا لتحدي أخر، وتبدأ عملية التصميم من جديد.
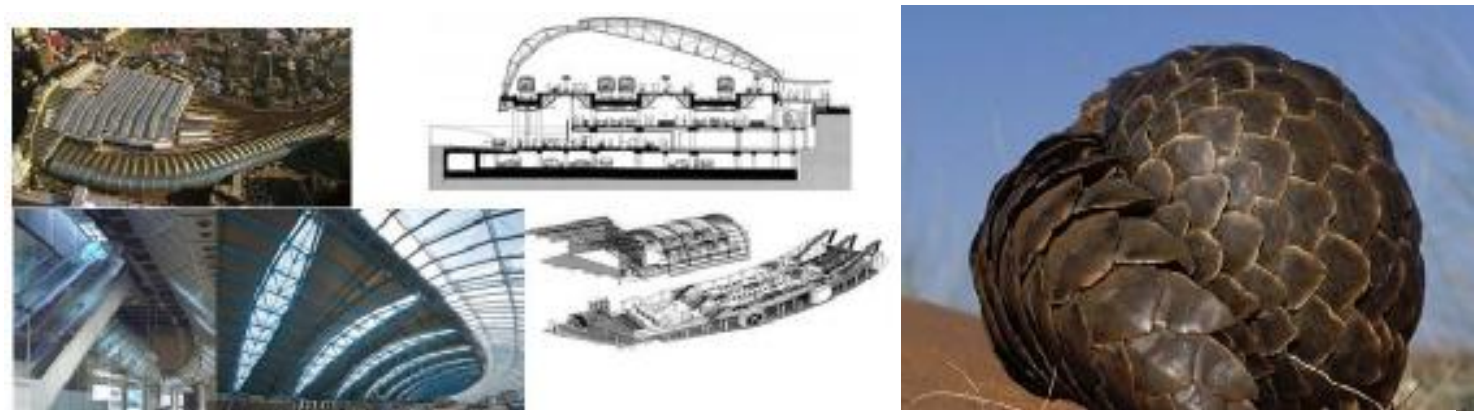

شكل (1) محاكاة محطة واترلو الدولية لحيوان المنغول 
جدول ( • 1) حلزونية التصميم بمحاكاة الطبيعة. المصدر: M.Benyus, 1997.

\begin{tabular}{|c|c|}
\hline & المرحلة \\
\hline 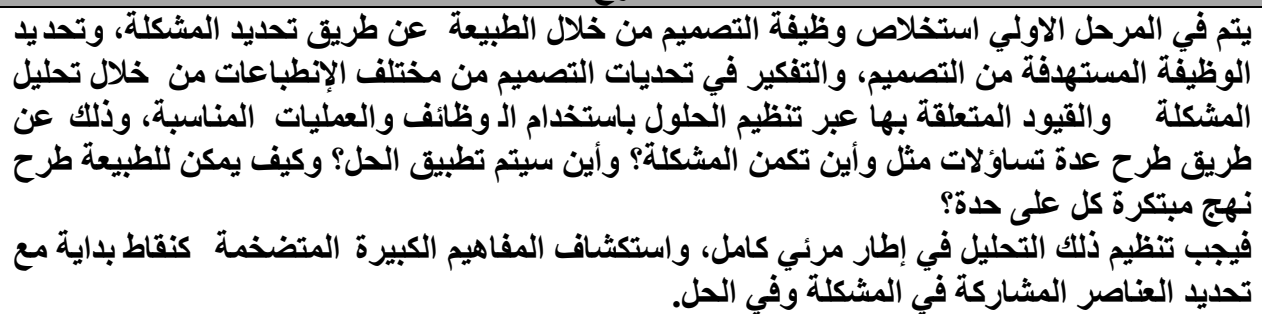 & ـ المرحلة الاولي: الاستخلاص \\
\hline 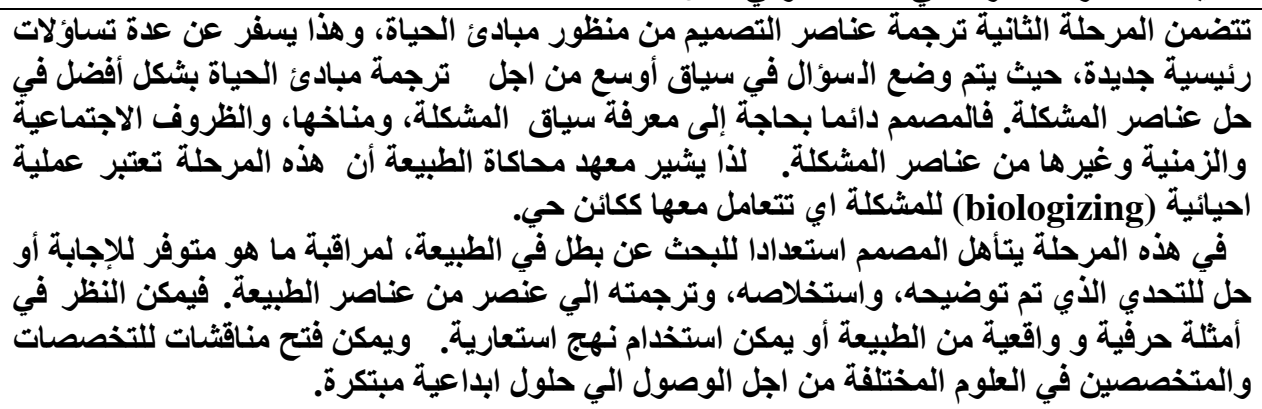 & $\begin{array}{l}\text { ـالمرحلة الثاتية: الترجمة } \\
\text { Translate } \\
\text { “LIFE TRANSLATE } \\
\text { PRINCIPALS INTO } \\
\text { DESIGN } \\
\text { PARAMETERS” }\end{array}$ \\
\hline 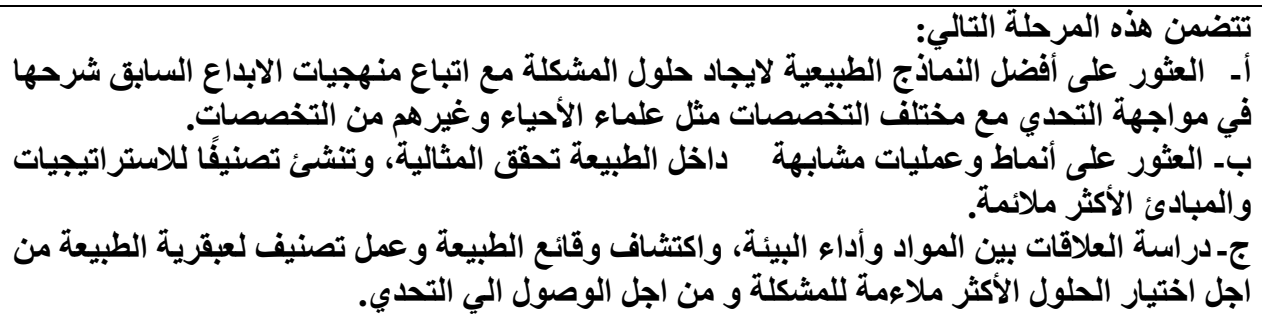 & $\begin{array}{r}\text { ـ المرحلة الثالثة: الاكتشافات } \\
\text { Discover } \\
\text { (اكتشافات انماط الطبيعةة) }\end{array}$ \\
\hline 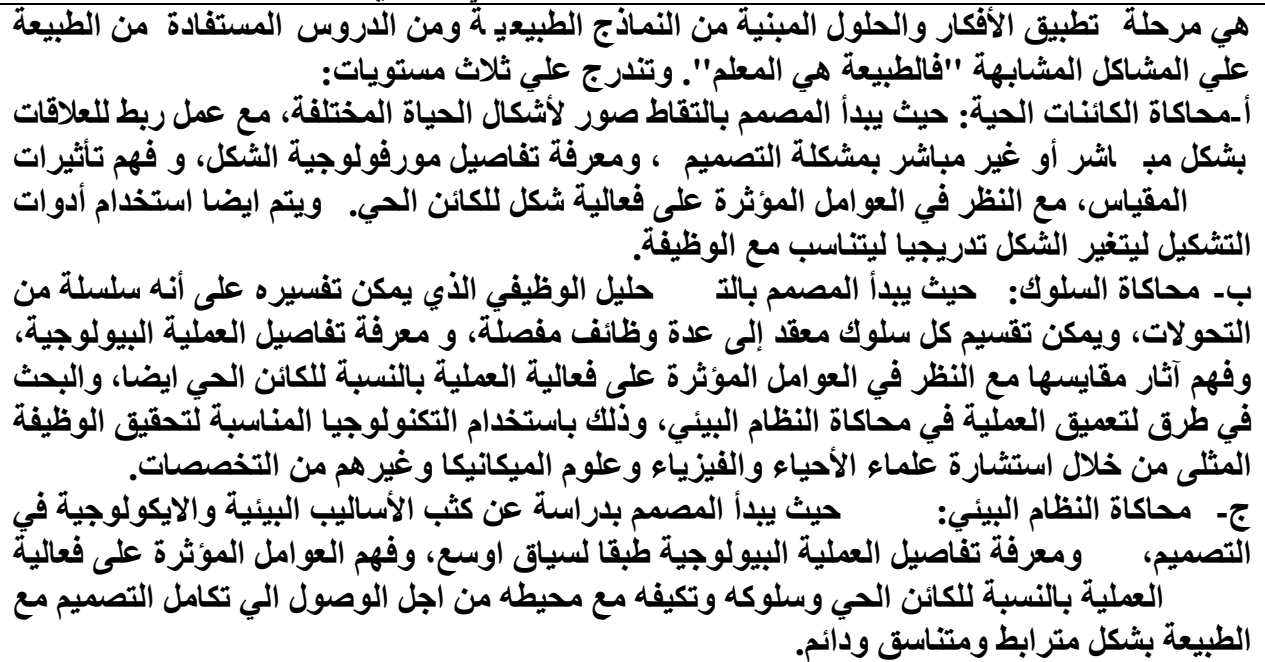 & $\begin{array}{r}\text { ـ المرحلة الرابعة: المحاكاة } \\
\text { Emulate } \\
\text { (محاكاة استراتيجيات الطبيعة) }\end{array}$ \\
\hline 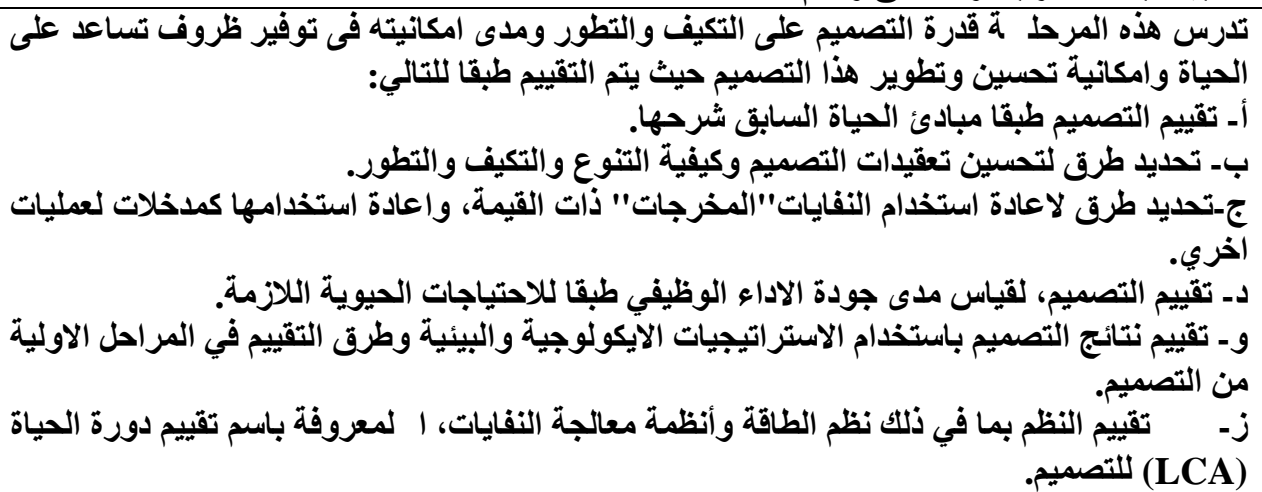 & $\begin{array}{r}\text { ـ المرحلة الخامسة: التقينيم } \\
\text { Evaluate }\end{array}$ \\
\hline
\end{tabular}




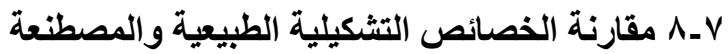

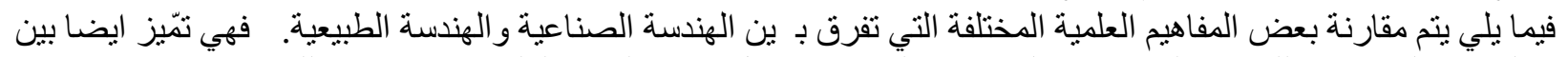

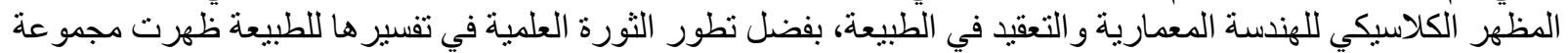

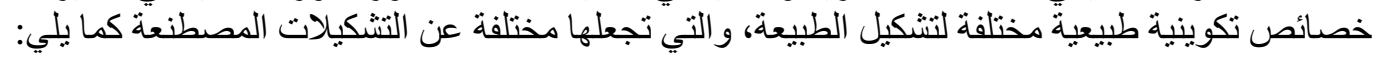

جدول (11) مقارنة الخصائص التثكيلية للطبيعة والخصائص التثكيلية المصطنعة. المصدر: الباحث

\begin{tabular}{|c|c|}
\hline الخصائص التشكيلية المصطنعة & الخصائص التشكيلية الطبيعية \\
\hline تتبع الهندسة الاقليدية & تتبع الهندسة الغير اقليدية \\
\hline التطابق الذاتي & التشابه الذاتي \\
\hline التكرار المتطابث & التكرار المتجانس "النستخ المتماثل" \\
\hline الاختلاف & التباين \\
\hline شكل ثُابت & شكل ديناميكي \\
\hline الإنتاج المتضخم & التعديل التكيفي الثـامل \\
\hline الصلابة & المرونة/القابلية للتطويع \\
\hline
\end{tabular}

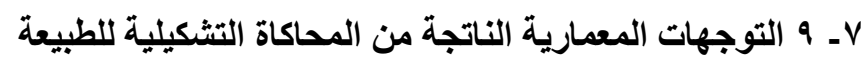

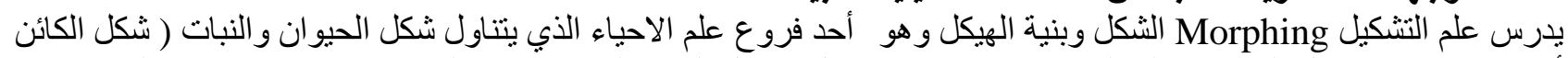

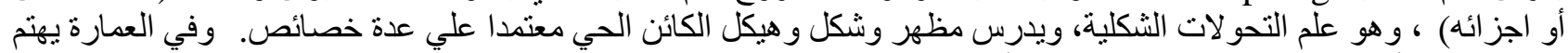

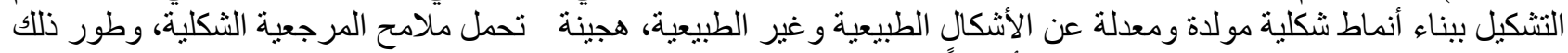

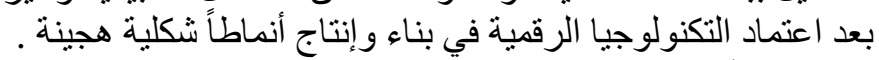

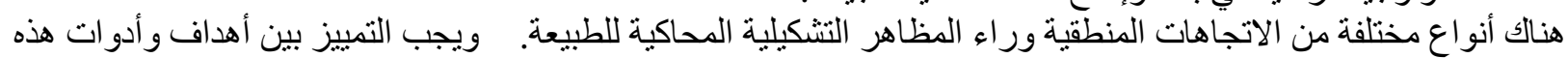
المظاهر ، حيث تتجلى الأهداف ما بين الثكل الجمالي ( اتجاه نحو الثكل) و التظهر الوظيفي ( الموجه نحو الأداء). و وهذا ما يتم

عرضه كالتالي:

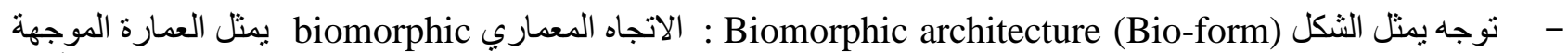

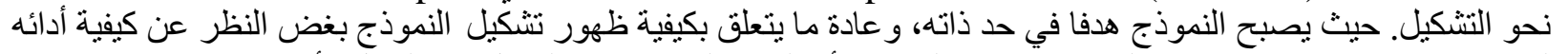

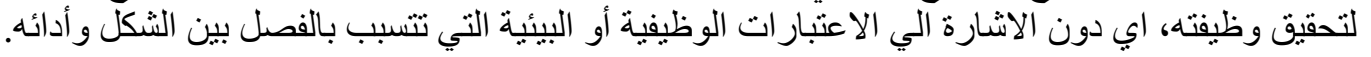

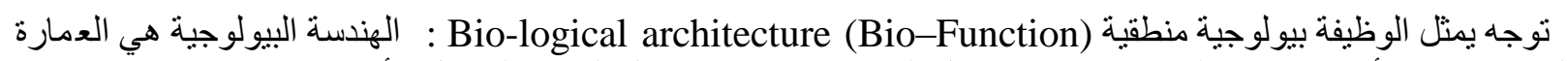
الموجهة نحو الأداء، ويهدف إلى إظهار مبادئ شكل الطبيعة فقط حيث تشئ تشكل المبادئ لتشغيل الأداء.

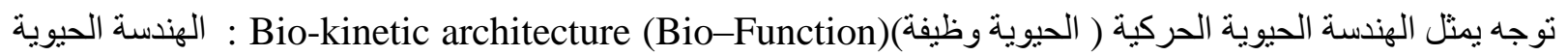
الذاتية الحركية هي عمارة موجهة نحو الأداءة الحئة وتهدف إلى إظهار مبادئ الحياة لتحقيق منطلبات الأداء.

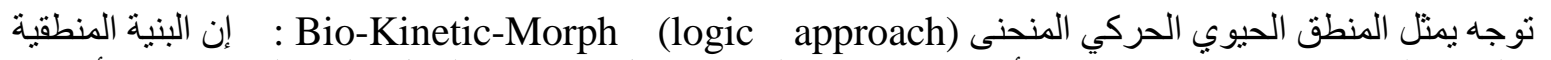

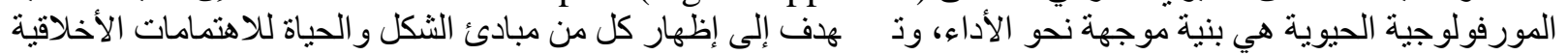
و الأداء. ويهتم بكل من شكل الطبيعة ومبادئ الحياة بنفس المقدارفي انشاء مباني تحقق أداء الطبيعة.

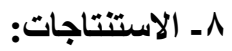

يمثل استخدام آلية "محاكاة الطبيعة" عامل مغاير مستحدث في حال تداخله في منظومة عمليات وّ"اليات العملية التصميمية

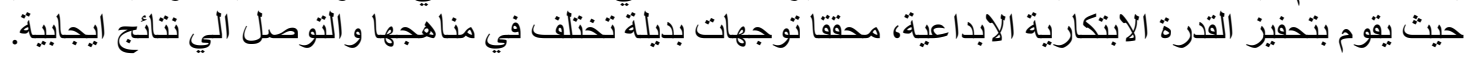

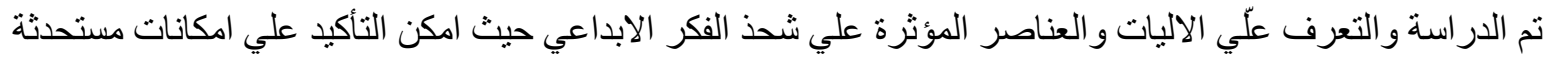

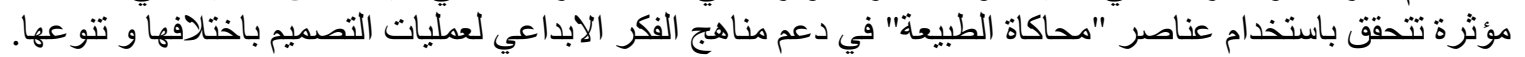

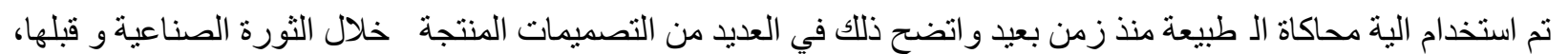

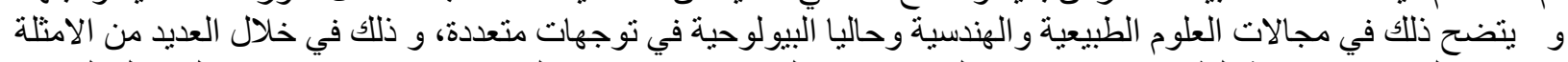

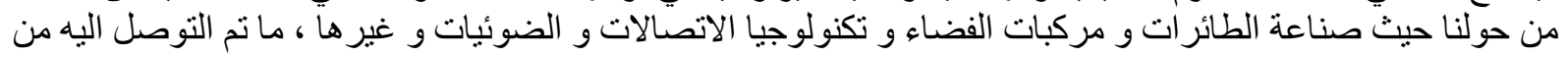

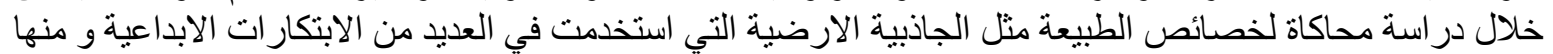
الصعود للفضاء.

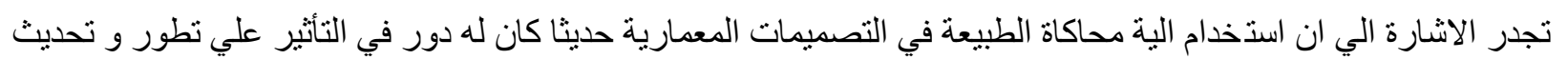

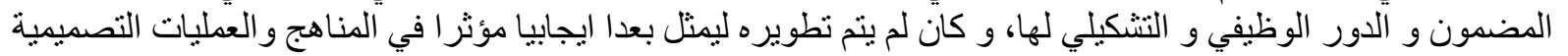

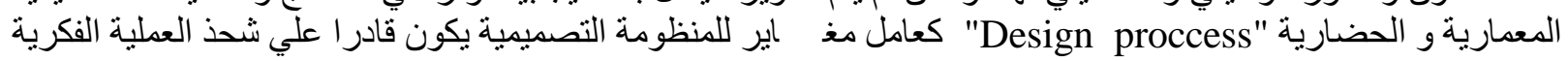




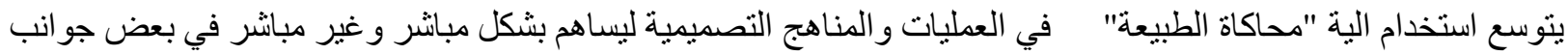
تحقيق الاتزان البيئي في نو احي متعددة للعمليات المرتبطة بالخصائص الطبيعية للمنتج التصميمي المستهدف.

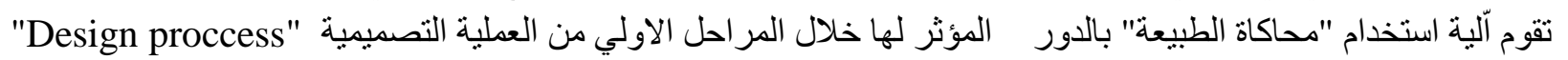

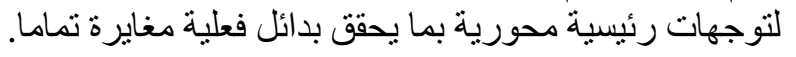

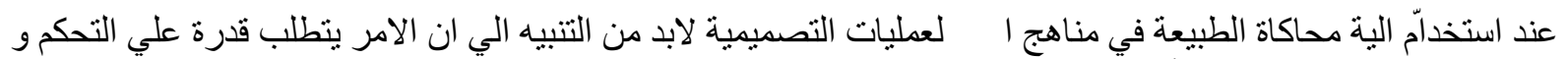

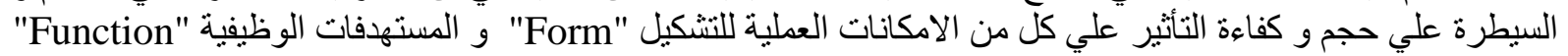

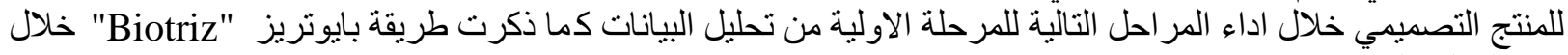

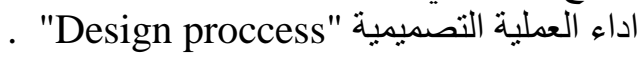

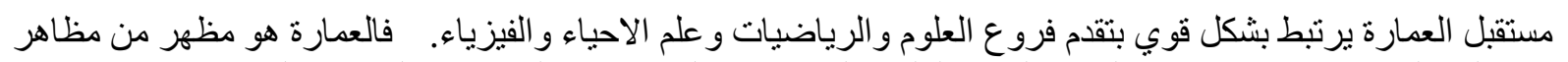

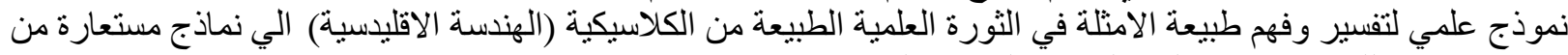
مظهاهر معقدة للطبيعة تعتمد علي (الهندسة الغير الاقليدسية).

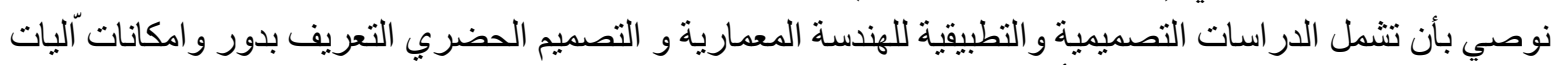

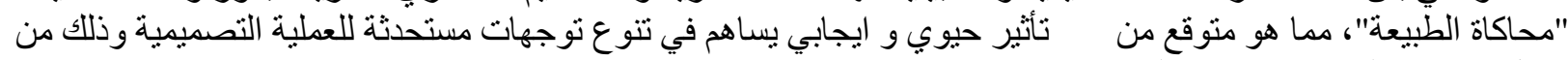

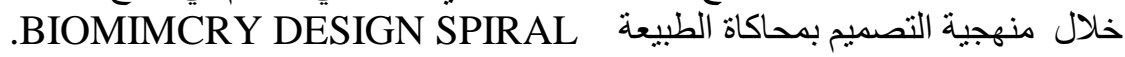

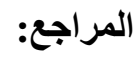

$$
\begin{aligned}
& \text { المراجع العربية: }
\end{aligned}
$$

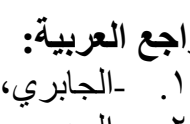

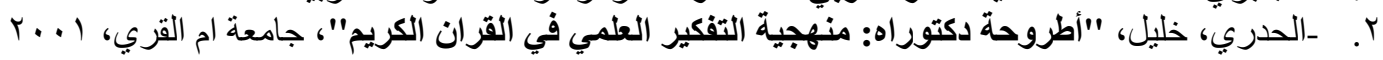

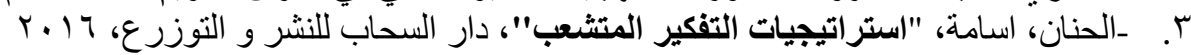

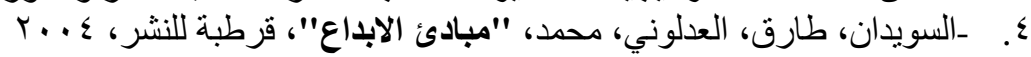

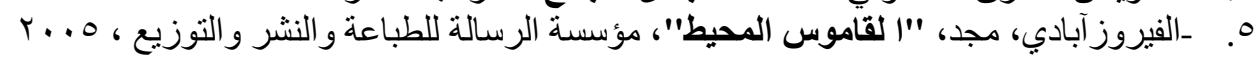

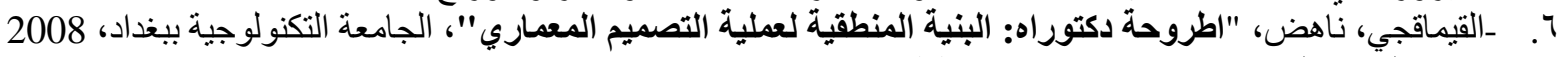

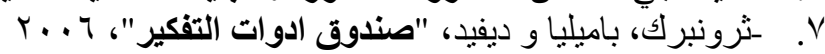

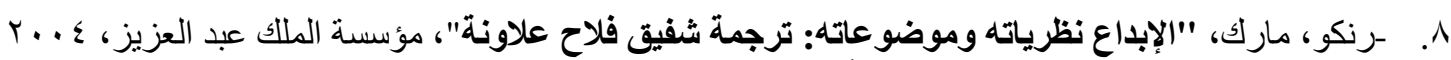

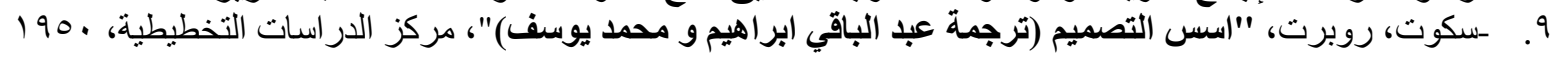

1. -Altshuller, henry, "The innovation algorithm: TRIZ, sys-tematic innovation and technical creativity", Technical Innovation Center, 1999, p 67:80

2. -Altshuller, henry, "\& Suddenly the Inventor Appeared: TRIZ, the Theory of Inventive Problem Solving, $2^{\text {nd }}$ ed.", Technical Innovation Center, 1996, p 5:55

3. BARAKAT, PA., BAKR, A., ELSAYAD, ZE., "TOWARDS A NEW BIOMIMIC APPROACH, NEW BIO-MIM-TRIZ DESIGN PROCESS", Proceedings of 34th IASTEM Int. Conf., Lon., 2016, P3:6

4. -Bullock, T.H., Bennett, M.V.L., Johnston, D., Josephson, R., Marder, E., "Fields

R.D.:The Neuron Doctrine, Redux, Science, V.310", 2005

5. -Darwin, Charles, "On the origin of species: By Means of nature selection", Dover Publications, 2006

6. -Davis, I., "Guide to RIBA Professional Services Contracts", RIBA Publishing, 2010

7. -De Bono, Ed., "Serious Creativity", Harper Business, 1992

8. H.Maslow, Abraham," A theory of Human Motivation and personality", Third Ed,New York, Harper, 1987.

9. -Jones, Christopher, "Design Methods: Seeks of human futures", Wiley-Interscience, 1970Wilkinson, I.M.S, "Essential Neurology", Blackwell publishing, 1993

10. -Laseau, P, "Graphic Thinking for Architects and Designers", Willey,1980

11. -M.Benyus, Janine, "Biomimcry: Innovation Inspired by Nature", Harper Perennial, 1997.

12. -McHarg, Ian, "Design with Nature”, Wiley, 1995. 
13. -Mehdi Kavandi, Alireza Jozepiri, Sharareh Teimouri and Fatemeh Abbasi Mehdi Sadri, "Bionic Architecture, Forms and Constructions", Research Journal of Recent Sciences, march 2014.

14. -Pawlyn, Michael, "Biomimicry in Architecture", RIBA, 2016.

15. Rush, Richard D, "The Building Systems Integration- Hand Book", the American Institute of

16. Architecture, John Willy \& Sons, New York, 1986.

17. -Weaver, Jonathan," Extending the TRIZ methodology to Connect Engineering Design Problems to Biological Solutions", Article for NCIIA 16th Annual Conference, 2015.

-https://www.thoughtco.com/the-idea-of-nature-2670631

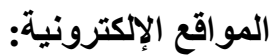

-https://plato.stanford.edu/entries/natphil-ren/

-https://biomimicry.org/

https://biomimicry.net/the-buzz/resources/designlens-lifes-principles/ 